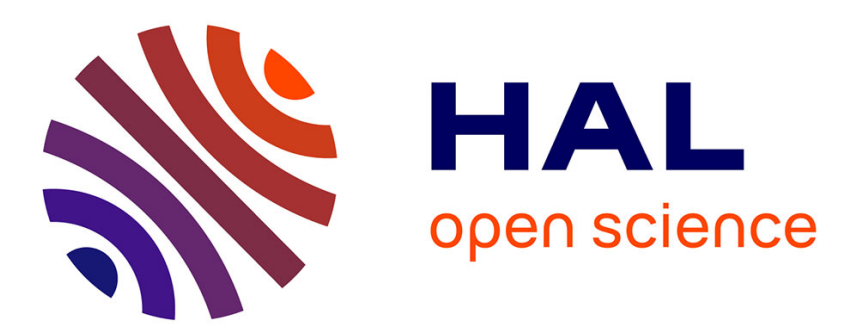

\title{
Why is the Energy of the Singly Occupied Orbital in Some Radicals below the Highest Occupied Orbital Energy?
}

Laura Abella, Jeanne Crassous, Ludovic Favereau, Jochen Autschbach

\section{To cite this version:}

Laura Abella, Jeanne Crassous, Ludovic Favereau, Jochen Autschbach. Why is the Energy of the Singly Occupied Orbital in Some Radicals below the Highest Occupied Orbital Energy?. Chemistry of Materials, 2021, 33 (10), pp.3678-3691. 10.1021/acs.chemmater.1c00683 . hal-03268336

\section{HAL Id: hal-03268336 \\ https://hal.science/hal-03268336}

Submitted on 29 Jun 2021

HAL is a multi-disciplinary open access archive for the deposit and dissemination of scientific research documents, whether they are published or not. The documents may come from teaching and research institutions in France or abroad, or from public or private research centers.
L'archive ouverte pluridisciplinaire HAL, est destinée au dépôt et à la diffusion de documents scientifiques de niveau recherche, publiés ou non, émanant des établissements d'enseignement et de recherche français ou étrangers, des laboratoires publics ou privés. 


\title{
Why is the energy of the singly occupied orbital in some radicals below the highest occupied orbital energy?
}

\author{
Laura Abella, ${ }^{a}$ Jeanne Crassous, ${ }^{b}$ Ludovic Favereau ${ }^{b}$, and Jochen Autschbach ${ }^{a, *}$ \\ ${ }^{a}$ Department of Chemistry, University at Buffalo, State University of New York, Buffalo, NY \\ 14260-3000, USA. Fax: (+) +01-716-645-6963. email: jochena@ buffalo.edu \\ ${ }^{b}$ Univ Rennes, CNRS, ISCR - UMR 6226, F-35000 Rennes, France
}

\begin{abstract}
Organic (mono-) radicals where the singly occupied molecular orbital (SOMO) is energetically below the highest occupied molecular orbital (HOMO) level have recently attracted much interest. A clear understanding of the electronic factors that lead to this energetic SOMO/HOMO inversion (SHI) would be desirable to aid the rational design of SHI radicals with high stability and other desired properties. The electronic factors that govern SHI in known SHI radicals are studied computationally. Then, the findings are applied to design potential SHI candidates. The electrostatic repulsion among the frontier orbitals, and the repulsion between the $\alpha$ and $\beta$ spin components of the orbitals in a closed-shell 'parent' compound, are key to understanding the occurrence of SHI in a radical.
\end{abstract}

\section{Introduction}

Open-shell electronic structures have long been a focus of research in chemistry and biochemistry. Persistent organic radicals are also of high interest in materials science, due to the possibility to combine electron and spin conduction in molecular electronics such as organic field-effect transistors (OFETs), organic light-emitting diodes (OLEDs), and organic magnets. ${ }^{[1] 7]}$ In particular, organic radical emitters with high luminescence and high photostability remain the target of intense efforts. They have recently attracted considerable attention owing to the doublet-spin character of their excited states, which can circumvent efficiency limitations imposed by electrically generated triplet excitons, making them exciting alternatives for the basis of next-generation optoelectronics. $\stackrel{8}{~ T h e ~ d e s i g n ~ o f ~ o r g a n i c ~ o p e n-s h e l l ~ m o l e c u l e s ~ r e m a i n s ~ a ~ f o r m i d a b l e ~ c h a l l e n g e ~ b e c a u s e ~ o f ~ t h e ~}$ usually high chemical reactivity and poor configurational stability of radicals.

Recently, the team of Li, Brédas \& Friend (LBF) and coworkers ${ }^{9}$ designed donor-acceptor neutral radicals based on the electron-poor groups perchlorotriphenylmethyl $\left(\mathrm{PTM}^{\bullet}\right)^{10}$ or tris $(2,4,6$ trichlorophenyl)methyl $\left(\mathrm{TTM}^{\cdot 0}\right), \frac{11}{11}$ combined with an electron-rich moiety, such as phenylphenothiazine (PPTA) or 9-(naphthalene-2-yl)-9H-carbazole (NCz). In combination, these groups form neutral radical systems. What is particularly interesting about these, and certain other (neutral or charged) radical systems reported in the recent literature, ${ }^{7 \sqrt{12} \sqrt{28}}$ is a ground-state electronic structure where the singly occupied molecular orbital (SOMO) associated with the radical charac- 
ter of the system is energetically below the highest (doubly) occupied molecular orbital (HOMO) level. This energetic SOMO-HOMO inversion (SHI) sets the electronic properties of the radical apart from the more common situation where the SOMO is the highest occupied spin orbital of the system. Accordingly, SHI has been associated with an unusual stabilization of rad-

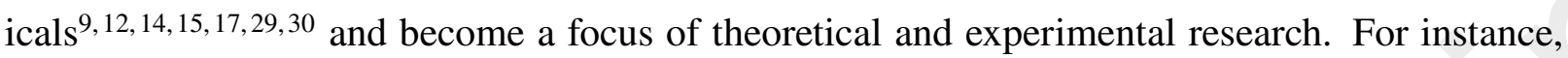
SHI radicals have been considered as luminescent emitters in OLED devices. 9 It is tempting to associate SHI, as some have done, with a non-aufbau electronic configuration. Violations of the aufbau rule have been noted previously for certain types of high-spin restricted open-shell Hartree-Fock (ROHF) calculations. ${ }^{31}$ In spin-unrestricted MO theory, that is, in spin-unrestricted HF or Kohn-Sham (KS) density functional theory (DFT) calculations, matching pairs of $\alpha$ spin $(\uparrow)$ and $\beta$ spin $(\downarrow)$ MOs may have different energies. For the reported SHI cases, some of which are discussed later as representative examples, the electron-hole (unoccupied) counterpart of the SOMO_which we label SUMO for convenience, with $\mathrm{S}=$ 'single' meaning its opposite-spin partner is in the occupied space-is above the HOMO in energy in such calculations. Therefore, there is no unoccupied spin orbital energetically below the highest occupied spin orbital. Nonetheless, of course, the occurrence of SHI in some radicals is very intriguing.

A clear explanation of the electronic factors that lead to SHI has not been forthcoming, but would be desirable in order to design SHI radicals with relatively high stability and other uncommon properties rationally, for instance, with the aid of computational pre-screening and 'big data' machine-learning approaches. Thus, in this study we focus on understanding the electronic factors that govern SHI in already reported radicals. The findings are then applied to confirm that computations are able to identify potential SHI candidates relatively straightforwardly. In addition to the precursors used by LBF and coworkers,, 916 leading to neutral SHI radicals, we study several cases where different chromophores are combined in a neutral closed-shell system, which is then oxidized and forms an SHI radical cation. Here, naphthalene diimides (NDIs) ${ }^{32}+34$ and thionated NDIs (TNDIs), $\sqrt[356]{36}$ known for their excellent thermal, light, and air stability, were selected for the electron deficient fragment and combined with common organic electron donor moieties. We also investigate the reasons for SHI occurring in aza-thia[7] helicene $\left(\mathrm{ATH}^{\bullet}\right), \frac{15}{15}$ a benzo-annulated tetrathiafulvalene derivative $\left(\mathrm{ETBN}^{\bullet}\right)^{0,37}$ and a chiral bicarbazole radical cation $\left(\mathrm{BCz}^{\bullet+}\right) ! 17$

A common theme emerges from the analysis: The formation of an SHI radical from a closedshell parent compound (i.e., with an even number of electrons) is likely to occur when the electrons in the highest molecular orbital (HOMO) of the closed-shell parent repel each other strongly, while at the same time the repulsion between the HOMO and other occupied frontier MOs is much weaker. The loss of a considerable amount of repulsive energy upon removing one of the HOMO occupations then drives the SHI radical formation. In the case of the bicarbazole radical cation, as a representative of a system composed of two identical fragments, the occurrence of SHI requires a 
significant distortion of the molecular structure upon oxidation, causing the HOMO and SOMO to localize of separate fragments. We then show how these findings can be used to inform the design of new SHI radicals. These design strategies are demonstrated to produce the desired results 'in silico'.

\section{Computational Details}

Molecular structure optimizations were performed with spin-restricted and spin-unrestricted (U) KS DFT as implemented in the Gaussian (G16) ${ }^{\sqrt[38]{3}}$ package, using the PBE0 functional ${ }^{\sqrt{39}}$ and the def2-SV(P) basis. ${ }^{40411}$ 'D3' dispersion corrections ${ }^{42}$ and solvent effects (via the polarizable continuum mode ${ }^{43 / 44}$ for dichloromethane, acetonitrile and cyclohexane, as applicable, to match experimental conditions) were included in the structure optimizations. Molecular orbital energies were analyzed with a locally modified version of NWChem, ${ }^{45 / 46}$ based on release 6.8 , also using UKS DFT with the PBE0 functional and the def2-SV(P) basis. The modified code is available on GitHub. ${ }^{47}$ Test calculations with other functionals were performed to ensure that the presence of SHI was not dependent on one particular choice of functional. Relevant data are provided in the Supporting Information (SI), Tables S15, S16 and S17. Moreover, test calculations without and with solvent effects, the latter being included in the NWChem calculations via the COnductor-like Screening MOdel' (COSMO), ${ }^{48}$ were performed to confirm that the SHI did not come about due to solvent effects (see SI for representative calculations including solvent effects). The MO energies from the gas phase calculations were then subjected to a detailed analysis as described in Section 3.1. Modifications of the NWChem code were made for the purpose of the present study, to generate the various contributions to the MO energies individually, along with selected electron repulsion integrals for the MOs.

\section{Results and Discussion}

\subsection{Analysis Protocol}

In UKS DFT, sets of $\operatorname{spin} \sigma=\alpha$ or $\beta$ MOs are determined in a coupled self-consistent field (SCF) cycle. The energy $\varepsilon_{i, \sigma}$ of MO number $i$ in spin set $\sigma$ is

$$
\varepsilon_{i, \sigma}=T_{i, \sigma}+V_{i, \sigma}^{\mathrm{nuc}}+V_{i, \sigma}^{C}+V_{i, \sigma}^{\mathrm{XC}}
$$

Here, $T_{i, \sigma}$ denotes the kinetic energy for the MO, $V_{i, \sigma}^{\text {nuc }}$ is the potential energy from the electronnucleus attraction, $V_{i, \sigma}^{C}$ is the potential energy from the Coulomb electrostatic repulsion due to all occupied MOs, and $V_{i, \sigma}^{\mathrm{XC}}$ is the orbital energy contribution from the exchange-correlation potential. 


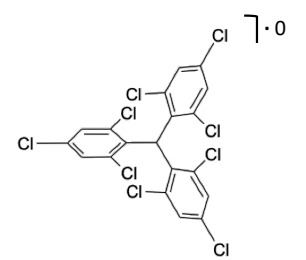

$\operatorname{TTM}^{\circ} 0$

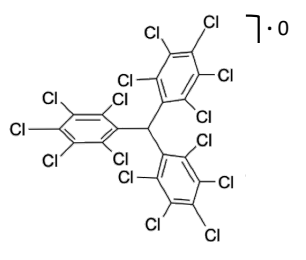

PTM $^{\circ} 0$

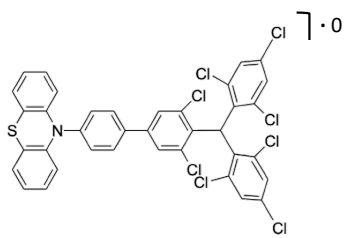

TTM-PPTA ${ }^{\circ}$

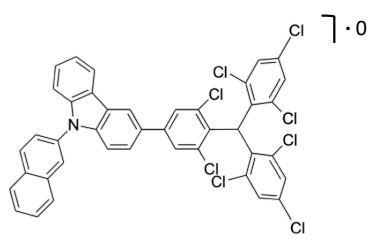

TTM-3CNz ${ }^{\circ}$

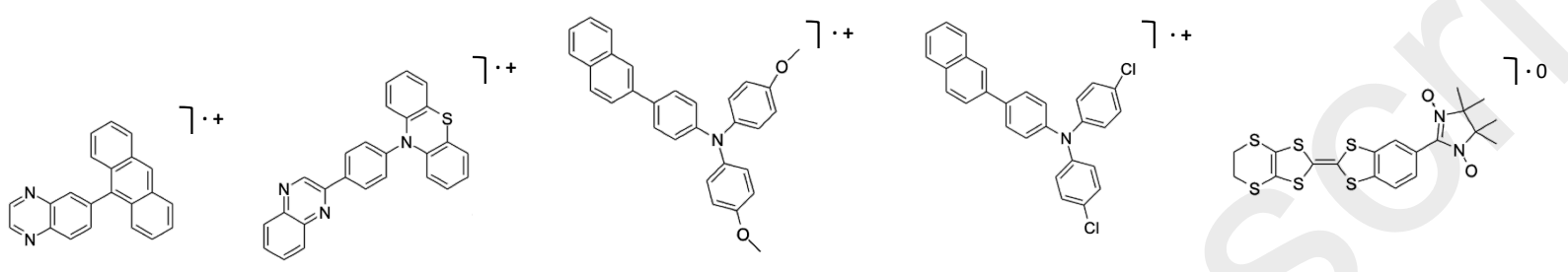

DAN-ANT ${ }^{+}$

DAN-PPTA ${ }^{-+}$

NAP-TAA-MeO +

NAP-TAA-Cl +

ETBN $^{\circ} 0$

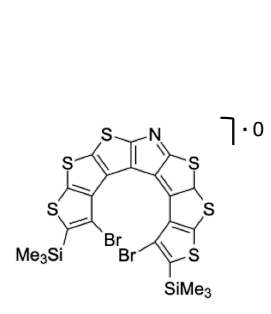

$\mathrm{ATH}^{\circ} 0$

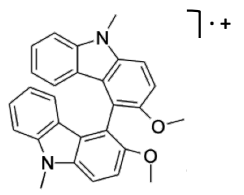

$\mathrm{BCz}^{+}$

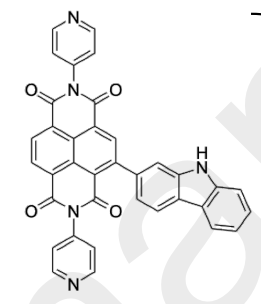

NDI-Mol-2 +
$7 \cdot+$

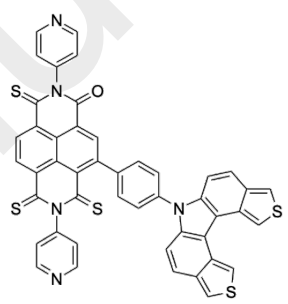

TNDI-Mol-3. +

Figure 1: Selected radical systems studied in this work: $\mathrm{TTM}^{\bullet}, \mathrm{PTM}^{\bullet}$, $\mathrm{TTM}^{-\mathrm{PPTA}^{*}}{ }^{0}$, $\mathrm{TTM}^{-}$

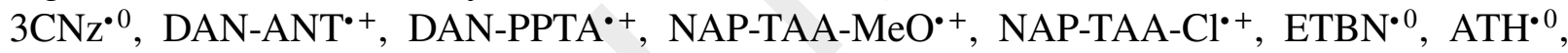
$\mathrm{BCz}^{\bullet+}$, NDI-Mol-2 ${ }^{+}$, and TNDI-Mol-3 ${ }^{\bullet+}$ The labels are explained in the text. In all cases, the closed-shell parent system considered in the analysis has an additional electron.

In the formally exact KS approach, and in calculations with most of the available approximate nonhybrid functionals, the XC potential is local. In generalized KS (GKS), such as in calculations with hybrid functionals, exact (Hartree-Fock-like) exchange is part of a non-local XC potential, and a range separation is often used for the exchange, for instance to improve calculated electronic spectra or band gaps of solids. Note that in SCF calculations such as (U)KS, the sum of the occupied MO energies counts the electron repulsion twice and is therefore not equal to the total energy.

The energy of the highest occupied spin $\mathrm{MO}, \varepsilon_{H}$, plays an important role in KS and GKS theory, in that it formally corresponds exactly to the negative vertical ionization potential (IP) of the system. .99 Thus, whether the SOMO of a radical is calculated to be below the highest occupied energy must have important consequences regarding the stability of the radical, or whether it can be turned into a bi-radical. In actual calculations, i.e., with approximate functionals, the numerical magnitudes of IP and $\varepsilon_{H}$ may differ. Nonetheless, if calculations with different functionals show a robust and large SHI, it is very likely that this correlates with the observed chemical properties. 
Approximate functionals with range-separated exchange can also be 'tuned' non-empirically ${ }^{50}$ to force $\varepsilon_{H}=-\mathrm{IP}$, which often also improves a range of other calculated molecular properties $51-53$ A downside of this type of tuning is that the range-separation parameters in the functional become system-dependent, and therefore not easily transferable between molecules. We opted to use the very well tested global hybrid PBE0 for the present calculations. Note, also, that this functional was deemed to be essentially free from empirically fitted parameters. $\underline{54}$

For the analysis, the formation of the radical is conceptually broken down as follows: We start with a closed-shell parent system in its equilibrium structure (CS//CS). The closed-shell parent has one more electron than the radical, but is otherwise equivalent. The CS//CS system is then ionized, but neither the MOs nor the molecular structure are yet allowed to respond to the loss of the electron ( $\tilde{R} / / C S)$. Next, the MOs relax, via SCF, to minimize the electronic energy of the radical in the CS molecular structure (R//CS). Finally, a geometry optimization and final SCF is performed to determine the MOs that minimize the energy of the radical in its own equilibrium structure (R//R). At each step, the energies of the MOs that become the SOMO and HOMO in the radical are analyzed according to Equation (1). We found that during the last step, from R//CS to $\mathrm{R} / \mathrm{R}$, representing vertical vs. adiabatic ionization, with the exception of the bicarbazole, no dramatic changes in the MO energies of interest take place in the studied systems. Rather, there are usually comparatively large, nearly equal but opposite, changes in $V_{i, \sigma}^{\text {nuc }}$ and $V_{i, \sigma}^{C}$ during this step. The changes in the MO energies that lead to SHI most often take place when going from CS//CS to R//CS. Therefore, the following discussion focuses on these steps for most of the studies cases. Figure 1 shows the radicals considered herein. Additional data for these systems, and results from calculations for additional molecules, are provided in the SI.

\subsection{Initial Assessment: TTM-PPTA $^{\circ}$ vs. TTM-3CNz ${ }^{0}$}

For illustration of the analysis, consider the $\mathrm{TTM}^{-\mathrm{PPTA}^{*}}{ }^{0}$ radical reported by the team of LBF and coworkers in Reference 9 . Figure 2 shows isosurface plots of the MOs, and the MO energies, for the CS//CS parent (the $\alpha$ and $\beta$ MOs are the same in this case, and not shown separately), $\mathrm{R} / / \mathrm{CS}$, and $\mathrm{R} / / \mathrm{R}$, from left to right. SHI of sizable magnitude, 0.5 to $0.6 \mathrm{eV}$, is readily visible for R//CS and R//R. [Orbital energies and energy gaps quoted in the text are for the $\alpha$ spin MOs, unless stated otherwise.] The SOMO of the radical is localized on the TTM fragment. A pair of matching occupied spin orbitals, with nearly identical appearance of the isosurfaces, is localized on the PPTA fragment and constitutes the HOMO. The SUMO is the lowest unoccupied spin $\mathrm{MO}$, and its isosurface plot is seen to match that of the SOMO near-perfectly, as already shown in Reference 9. [This is not always the case. For example, in previous, unrelated, studies $55-57$ we encountered radicals where the $\beta$ spin SUMO represented the electron hole in the calculated 


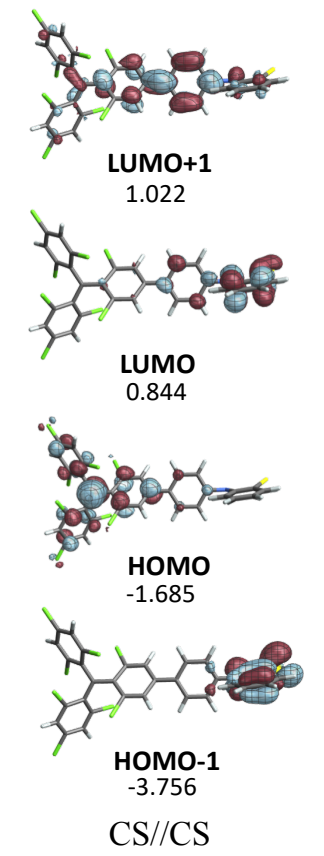

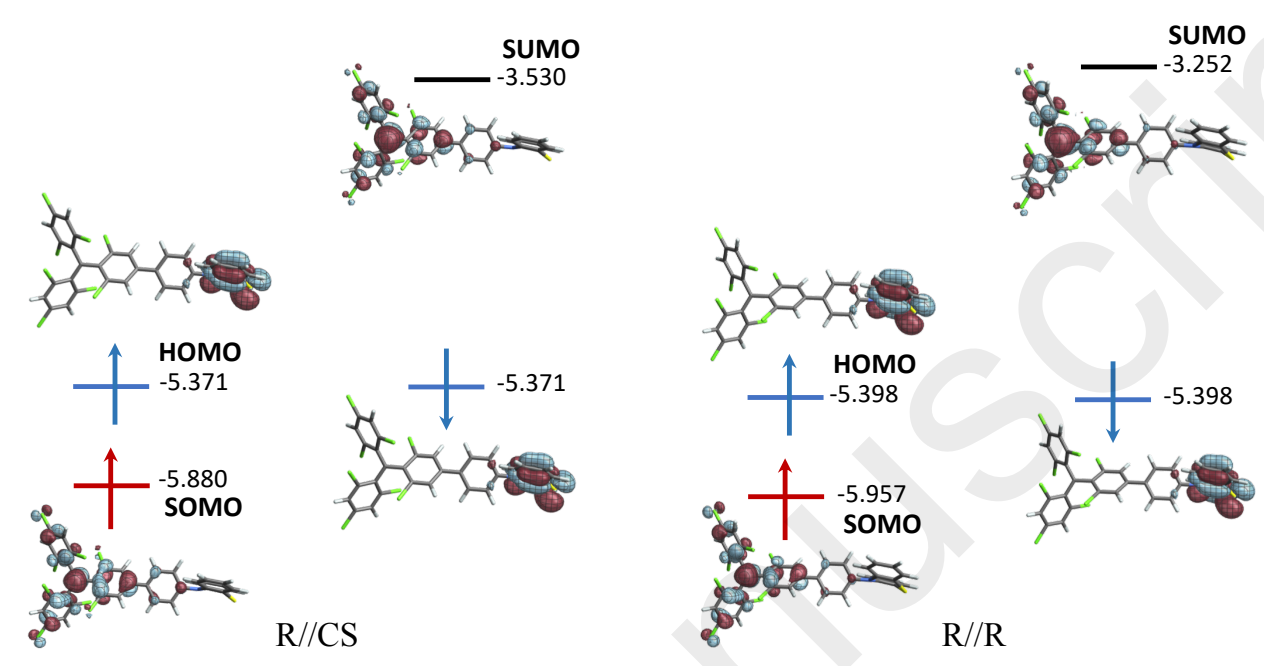

$\alpha \quad \beta$

Figure 2: Orbital energies (in eV) and isosurfaces ( \pm 0.030 a.u.) of selected MOs of the TTMPPTA system. See Section 3.1 for a description of the labeling.

spin density very well, but there was no $\alpha$ spin orbital that clearly matched the appearance of the SUMO. However, a linear combination of the occupied $\alpha$ spin MOs may create a matching SOMO, with a corresponding averaged orbital energy. An example is discussed later.] Inspection of the HOMO and HOMO-1 of CS//CS shows that there is an energetic re-ordering of the occupied spin MOs taking place when going from the closed-shell parent to the radical. While the energies of both the HOMO and HOMO-1 of CS drop significantly upon ionization, the HOMO of CS drops far more, leading to the SHI in the radical. It is reassuring that the ionization takes place from the HOMO of the closed-shell parent, as it must. Subsequent ionization of the neutral radical would involve the radical HOMO (see Figure S2), thereby leading to the formation of a bi-radical cation rather than a closed-shell cation (Figure 3).

Table 1 provides the breakdown of the MO energies of the TTM-PPTA system, following the conceptual steps of the radical formation introduced earlier. The $\stackrel{\Delta}{\rightarrow}$ data columns provide the changes along each step. The upper set of data track the energy of the CS HOMO becoming the SOMO of the radical, while the lower set of data track the energy of the CS HOMO-1 becoming the $\alpha$ spin component of the HOMO level of the radical. The large drop in energy for HOMO $\rightarrow$ $\mathrm{SOMO},-4.3 \mathrm{eV}$ overall, is for the most part associated with a -6.0 change in $V^{C}$ in the initial $\mathrm{CS} / / \mathrm{CS} \rightarrow \tilde{\mathrm{R}} / / \mathrm{CS}$ step. When the MOs relax in the $\tilde{\mathrm{R}} / / \mathrm{CS} \rightarrow \mathrm{R} / / \mathrm{CS}$ step, we see large changes in the kinetic energy and the various potential energy contributions, but they mostly cancel. The 


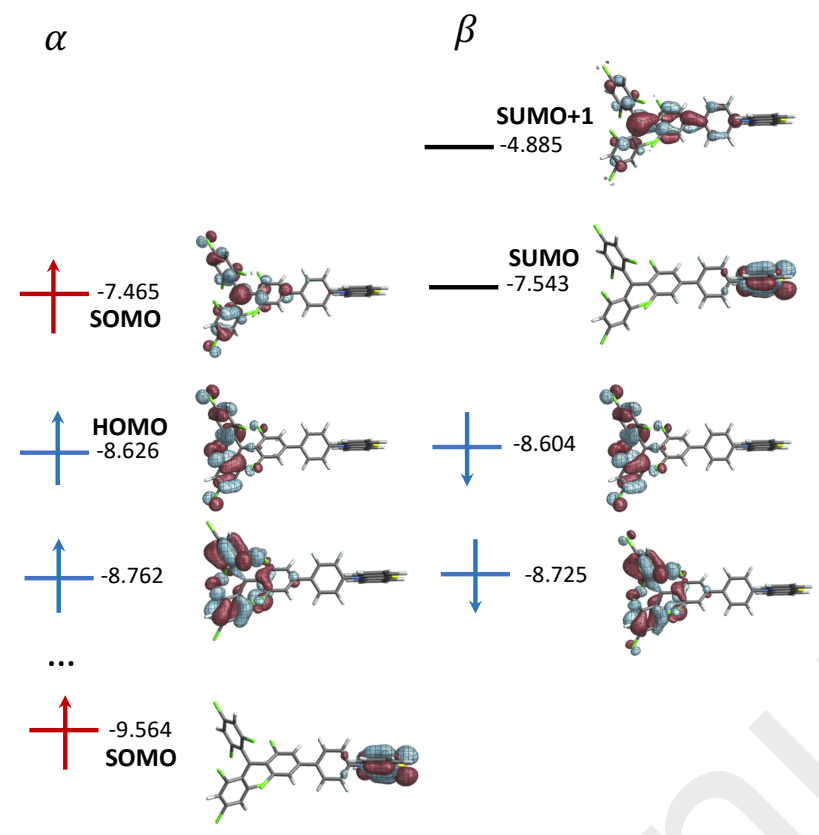

Figure 3: Orbital energies (in eV) and isosurfaces of selected molecular orbitals (MOs) ( \pm 0.030 au) for the bi-radical cation TTM-PPTA system.

occurrence of the SHI and its energetic magnitude are established at this point, and the subsequent

Table 1: MO energy contributions (in $\mathrm{eV}$ ) for the TTM-PPTA system. ${ }^{(a)}$ The numbers are for the $\alpha$ spin orbitals. See also Figure 2 .

\begin{tabular}{|c|c|c|c|c|c|c|c|}
\hline $\mathrm{HOMO} \rightarrow \mathrm{SOMO}$ & $\mathrm{CS} / / \mathrm{CS}$ & $\stackrel{\Delta}{\rightarrow}$ & $\tilde{\mathrm{R}} / / \mathrm{CS}$ & $\stackrel{\Delta}{\rightarrow}$ & $\mathrm{R} / / \mathrm{CS}$ & $\stackrel{\Delta}{\rightarrow}$ & $\mathrm{R} / / \mathrm{R}$ \\
\hline$T$ & 39.35 & 0.00 & 39.35 & 2.45 & 41.80 & 0.37 & 42.17 \\
\hline$-1320^{(b)}$ & -35.30 & 0.00 & -35.30 & 16.21 & -19.09 & -11.18 & -30.27 \\
\hline$+1320^{(b)}$ & 9.47 & -6.03 & 3.44 & -16.24 & -12.80 & 10.90 & -1.91 \\
\hline$V^{\mathrm{XC}}$ & -15.20 & 0.03 & -15.17 & -0.62 & -15.78 & -0.17 & -15.95 \\
\hline total $\varepsilon$ & -1.69 & -6.00 & -7.68 & 1.80 & -5.88 & -0.08 & -5.96 \\
\hline HOMO-1 $\rightarrow$ HOMO & $\mathrm{CS} / / \mathrm{CS}$ & $\stackrel{\Delta}{\rightarrow}$ & $\tilde{\mathrm{R}} / / \mathrm{CS}$ & $\stackrel{\Delta}{\rightarrow}$ & $\mathrm{R} / / \mathrm{CS}$ & $\stackrel{\Delta}{\rightarrow}$ & $\mathrm{R} / / \mathrm{R}$ \\
\hline$T$ & 45.46 & 0.00 & 45.46 & 0.22 & 45.68 & 0.04 & 45.73 \\
\hline$-1060^{(c)}$ & -20.61 & 0.00 & -20.61 & 4.07 & -16.54 & -3.44 & -19.98 \\
\hline$+1060^{(c)}$ & -12.55 & -1.42 & -13.97 & -4.54 & -18.51 & 3.40 & -15.11 \\
\hline$V^{\mathrm{XC}}$ & -16.06 & 0.00 & -16.06 & 0.06 & -16.00 & -0.03 & -16.03 \\
\hline total $\varepsilon$ & -3.76 & -1.42 & -5.18 & -0.19 & -5.37 & -0.03 & -5.40 \\
\hline
\end{tabular}

(a) CS: Closed-shell system. R̃: Radical without SCF, using the MOs of the closed-shell system. R:

Radical system. ${ }^{(b),(c)}$ The listed number was subtracted from $V^{\text {nuc }} / V^{C}$ to avoid large values in the other Table columns. Add it to get the full value of $V^{\text {nuc }} / V^{C}$. 
relaxation of the radical's structure and MOs, representing vertical vs. adiabatic ionization, does not alter the overall picture. Table 1 also shows that the HOMO of the radical has a much smaller change of $V^{C}$ in the initial CS//CS $\rightarrow \tilde{\mathrm{R}} / / \mathrm{CS}$ step, only $-1.4 \mathrm{eV}$.

Figure S6 and Table S1 in the SI show that the energetics of the HOMO $\rightarrow$ SOMO step are essentially the same for TTM-PPTA vs. the isolated closed shell TTM carbanion and the neutral radical, with a large change, $-6.4 \mathrm{eV}$ in $V^{C}$ for $\mathrm{HOMO} \rightarrow \mathrm{SOMO}$ in the initial CS//CS $\rightarrow \tilde{\mathrm{R}} / / \mathrm{CS}$ step, followed by an overall $1.8 \mathrm{eV}$ increase of the MO's energy in the subsequent orbital relaxation step. Due to the absence of another moiety with a higher energy fragment orbital, the $\mathrm{TTM}^{\bullet} 0$ radical's SOMO is the highest occupied orbital. Additional data for $\mathrm{PTM}^{\circ}$ are provided in the SI and show that it behaves qualitatively the same as $\mathrm{TTM}^{\circ}{ }^{0}$. We therefore do not discuss the systems involving $\mathrm{PTM}^{\bullet} 0$ in detail. Synthetically, the $\mathrm{TTM}^{\bullet} 0$ and $\mathrm{PTM}^{\bullet}$ radicals are prepared by first forming stable carbanions, followed by chemical oxidation. 10 [58|59 Indeed, the negative HOMO energy of the TTM/PTM carbanion, $-1.4 /-2.0 \mathrm{eV}$ in our calculations, indicates that it is stable, but not very difficult to ionize. The large negative SOMO energy of the $\mathrm{TTM}^{\bullet}{ }^{0} / \mathrm{PTM}^{\bullet} 0$ radical, $-6.0 /-6.5 \mathrm{eV}$, indicates that it would be very hard to ionize further by chemical means. This is of course also borne out by the oxidation potentials of the radicals, ${ }^{9}$ which were used by LBF and coworkers, along with the oxidation potentials of PPTA and other donor units, to rationalize the presence or absence of SHI in the combined system.

Table 2 provides selected electron repulsion integrals (ERIs) for the CS parent of TTM$\operatorname{PPTA}^{*}$. When the CS parent is ionized, the Coulomb repulsion between the $\alpha$ and $\beta$ spin counterpart of the HOMO disappears. The corresponding drop in $V^{C}$ for the remaining occupied $\alpha$ spin MO, before relaxation, therefore corresponds exactly to the $6.0 \mathrm{eV}$ 'self-Coulomb' ERI of the CS HOMO. The XC contributions are negligible in comparison. The large difference in the energies of the SOMO and the SUMO of the radical also has its origin in this large Coulomb ERI, because the energy of the SUMO still contains this term whereas the SOMO energy does not. The initial drop of the CS HOMO-1 energy upon ionization corresponds to the magnitude of the Coulomb ERI between HOMO and HOMO-1. The latter is only $1.4 \mathrm{eV}$, for the reason that the two MOs

Table 2: Selected Coulomb and exchange electron repulsion integrals (ERIs, in eV) for the closedshell parent systems of TTM-PPTA ${ }^{\circ}$ and TTM- $3 \mathrm{CNz}^{\circ}$.

\begin{tabular}{lrr}
\hline ERI Type & TTM-PPTA & TTM-3NCz \\
\hline self-Coulomb $^{(a)}$ HOMO-1 & 6.261 & 4.288 \\
Coulomb HOMO-1 / HOMO $^{(a)}$ HOMO & 1.422 & 4.180 \\
self-Coulomb & 6.029 & 6.147 \\
exchange HOMO-1 / HOMO & 0.001 & 0.363 \\
\hline
\end{tabular}

(a) This corresponds to the Coulomb repulsion between the $\alpha$ spin and the $\beta$ spin component of the MO. 


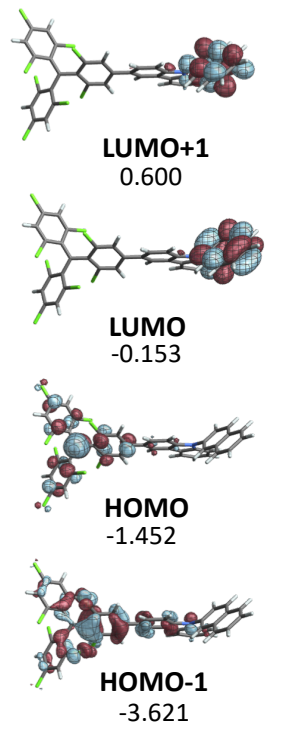

$\mathrm{CS} / / \mathrm{CS}$

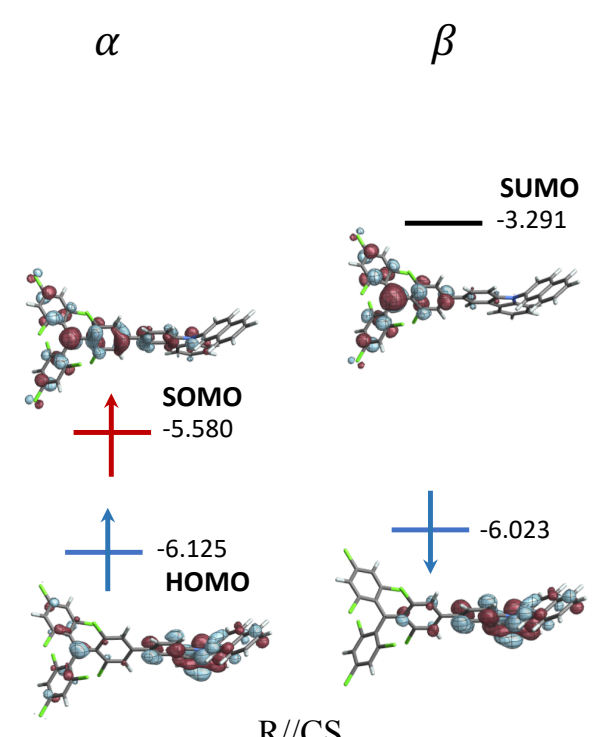

$\mathrm{R} / / \mathrm{CS}$

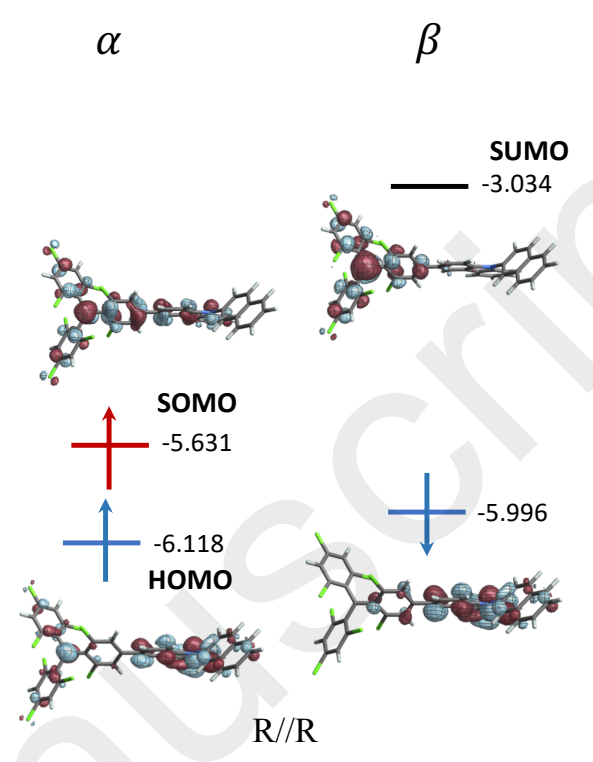

Figure 4: Orbital energies (in eV) and isosurfaces ( \pm 0.030 a.u.) of selected MOs of the TTM$3 \mathrm{NCz}$ system. See Section 3.1 for a description of the labeling.

in question are spatially disjoint, i.e., they hardly overlap. Spatial overlap of this kind between two MOs $\phi_{i}$ and $\phi_{j}$ can be quantified numerically, for example, via $\int\left|\phi_{i} \phi_{j}\right| d V$, which gives a result between 0 and 1 . However, the visualizations are already sufficiently clear in this regard. In other words, the disjoint MOs are centered on different sets of atoms. This is also indicated by the very small exchange ERI between HOMO-1 and HOMO. [The 'spatial overlap' should not be identified as a lack of orthogonality; the MOs are of course orthogonal.]

For comparison, consider TTM-3CNz ${ }^{\circ}$, which is another system that was reported by LBF and coworkers. It does not exhibit SHI. Figure 4 indicates that the HOMO and HOMO-1 of the CS parent overlap significantly, which is reflected in a large Coulomb ERI (4.2 eV) and a sizable exchange ERI $(0.4 \mathrm{eV})$ between the two orbitals (Table 2). The CS HOMO is comparable to that of TTM-PPTA, and TTM-centered, which results also in a comparable and large self-Coulomb ERI, $6.1 \mathrm{eV}$ in the case of TTM-3NCz. The CS HOMO-1 is not localized but spans both fragments. Upon ionization, the SUMO very closely resembles the HOMO of the closed-shell parent. The SOMO, on the other hand, shows delocalization between the two molecular fragments, not unlike the HOMO-1 of the closed-shell parent. We therefore see a significant degree of MO relaxation upon ionization, such that the SCF HOMO of R//CS is rather different from the HOMO-1 of $\mathrm{CS} / / \mathrm{CS}$. This relaxation is likely a result of the large Coulomb repulsion between the HOMO and HOMO-1 in the closed-shell system.

The MO energy changes in the CS//CS $\rightarrow$ R//CS step (where only the MO occupations differ, 
not the MOs themselves) give away the main reason why TTM-3CNz ${ }^{0}$ does not exhibit SHI. Again, the changes of the frontier MO energies in this step correspond overwhelmingly to the Coulomb ERIs involving the CS HOMO. There is a gap of over $2 \mathrm{eV}$ between HOMO and HOMO1 for the closed-shell parents of both TTM-PPTA and TTM-3NCz. This means the changes in the orbital energies upon ionization, not prior differences in the MO energies, determine whether SHI occurs in the two systems. For HOMO $\rightarrow$ SOMO, the MO energy change in the initial step, and all the way to R//R, is very similar between TTM-PPTA and TTM-3NCz. However, because of the much larger Coulomb ERI between the closed-shell HOMO and HOMO-1 in TTM-3NCz, the HOMO-1 also undergoes a very large drop in energy upon ionization, such that the resulting SOMO remains the highest occupied orbital. The subsequent orbital relaxation does not change this outcome.

It is worth-noting that LBF and coworkers showed that the presence of SHI in TTM-PPTA 0 system goes along with an enhanced fluorescence quantum yield and improved photostability, compared to TTM- $3 \mathrm{CNz}^{\cdot 0}$ which does not have SHI. ${ }^{9}$ Structural parameters of the DFT-optimized TTM-PPTA $^{\circ}$ and TTM-3CNz ${ }^{0}$ geometries have been analyzed, focusing on the angles and dihedral angles that connect the bridge between the TTM and, PPTA vs. 3CNz moieties. Both structures display comparable relative orientations between these moieties (Figure [55). A strong energetic stabilization from delocalization in the combined system is likely to drive $\pi$-system co-

Table 3: MO energy contributions (in eV) for the TTM-3NCz system. ${ }^{(a)}$ The numbers are for the $\alpha$ spin orbitals. See also Figure 4.

\begin{tabular}{|c|c|c|c|c|c|c|c|}
\hline $\mathrm{HOMO} \rightarrow \mathrm{SOMO}$ & $\mathrm{CS} / / \mathrm{CS}$ & $\stackrel{\Delta}{\rightarrow}$ & $\tilde{\mathrm{R}} / / \mathrm{CS}$ & $\stackrel{\Delta}{\rightarrow}$ & $\mathrm{R} / / \mathrm{CS}$ & $\stackrel{\Delta}{\rightarrow}$ & $\mathrm{R} / / \mathrm{R}$ \\
\hline$T$ & 39.55 & 0.00 & 39.55 & 1.91 & 41.46 & -0.10 & 41.36 \\
\hline$-1320^{(b)}$ & -47.11 & 0.00 & -47.11 & 51.34 & 4.23 & -0.52 & 3.71 \\
\hline$+1320^{(b)}$ & 21.36 & -6.15 & 15.21 & -50.70 & -35.49 & 0.66 & -34.83 \\
\hline$V^{\mathrm{XC}}$ & -15.25 & 0.03 & -15.22 & -0.56 & -15.78 & -0.09 & -15.87 \\
\hline total $\varepsilon$ & -1.45 & -6.11 & -7.57 & 1.99 & -5.58 & -0.05 & -5.63 \\
\hline HOMO-1 $\rightarrow$ HOMO & $\mathrm{CS} / / \mathrm{CS}$ & $\stackrel{\Delta}{\rightarrow}$ & $\tilde{\mathrm{R}} / / \mathrm{CS}$ & $\stackrel{\Delta}{\rightarrow}$ & $\mathrm{R} / / \mathrm{CS}$ & $\stackrel{\Delta}{\rightarrow}$ & $\mathrm{R} / / \mathrm{R}$ \\
\hline$T$ & 43.91 & 0.00 & 43.91 & -4.22 & 39.69 & 0.38 & 40.07 \\
\hline$-1250^{(c)}$ & -70.87 & 0.00 & -70.87 & 156.09 & 85.22 & -23.03 & 62.19 \\
\hline$+1250^{(c)}$ & 39.32 & -4.18 & 35.14 & -150.45 & -115.32 & 22.74 & 92.57 \\
\hline$V^{\mathrm{XC}}$ & -15.98 & 0.01 & -15.97 & 0.26 & -15.72 & -0.09 & -15.81 \\
\hline total $\varepsilon$ & -3.62 & -4.17 & -7.79 & 1.67 & -6.12 & 0.01 & -6.12 \\
\hline
\end{tabular}

(a) CS: Closed-shell system. R̃: Radical without SCF, using the MOs of the closed-shell system. R:

Radical system. There is strong orbital relaxation between $\tilde{R}$ and R. ${ }^{(b),(c)}$ The listed number was subtracted from $V^{\text {nuc }} / V^{C}$ to avoid large values in the other Table columns. Add it to get the full value of $V^{\text {nuc }} / V^{C}$. 
planarity, which then may lead to the loss of SHI. In such cases, keeping the different $\pi$ systems orthogonal, for example with the help of bulky substituent, ofers a way to restore SHI (see also Sec. 3.3). However, the comparable relative orientations of the donor and acceptor moieties in TTM$\mathrm{PPTA}^{\cdot 0}$ vs. TTM- $3 \mathrm{CNz}^{\circ}$ suggest that the MO localization in the former system is not forced by structural parameters.

\subsection{SHI by Design}

Considering the results obtained thus far, from the perspective of a closed-shell parent system, it appears that SHI will likely occur under the following conditions as determined in KS DFT calculations: (i) The HOMO and HOMO-1 of the closed-shell parent system do not overlap strongly, spatially, such that the Coulomb ERI between them is not very large. (ii) The energy gap between HOMO and HOMO-1 in the CS system should not be large, such that the energetic crossover upon ionization is facilitated. (iii) The orbital that becomes the SOMO in the radical should have a strongly stabilizing $V^{\text {nuc }}$ and be spatially relatively compact, such that it also has a large selfCoulomb repulsion when it is doubly occupied. In effect, then, the removal of an occupation in the HOMO goes along with a strong stabilization of the orbital itself, while at the same time the HOMO-1 undergoes a much weaker stabilization. The large self-Coulomb repulsion of this orbital, and the lack thereof in the radical, must also be considered the driving force in the total energy that causes the SHI electronic structure to represent the ground state of the radical.

Note that the existence of a stable radical precursor, neutral or charged, is not required for SHI to occur. For example, in a recent study ${ }^{17}$ we prepared chiral closed-shell bicarbazole derivatives that exhibited SHI upon chemical and electrochemical oxidation, as indicated by calculations and experimental data. Rajca et al. obtained an aza-thia[7]helicene and its distonic radical cation ${ }^{15}$ via chemical and electrochemical oxidations and demonstrated SHI in these systems. Other studies of SHI compounds, for instance TEMPO ${ }^{12}$ and nitronyl nitroxide $\mathrm{e}^{7]}$ stable radicals used chemical oxidation. Armed with these insights, we attempted a naive computational design of an SHI system, with simple chromophores as precursors for the fragments. We initially turned our attention to a molecular material based on anthracene (ANT) and 1,4-diazanaphthalene (DAN) units, giving their synthetic accessibility and their successful use in many optoelectronics applications (OLEDs, OFETs and organic PV). Moreover, DAN was selected because of the presence of the more electronegative nitrogen atoms which decreases the energy of its HOMO level in comparison to the ANT unit. The data in Figure S10 in the SI show that if extensive $\pi$ conjugation between the fragments can be avoided (per the discussion near the end of Sec. 3.2), for instance with the help of bulky substituents that keep the $\pi$ systems close to perpendicular, then the radical cation of a linked (C-C-bridged) DAN-ANT ${ }^{*+}$ system would exhibit SHI. The HOMO-SOMO energy 
gap in the radical is small, however, about $0.1 \mathrm{eV}$, which shows that there is much room for improvement. Such computational screening would also need to be followed up by 'reality checks' with synthetic chemists, of course, to see whether an SHI radical designed by computations can be synthesized in the laboratory.

As far as improvements are concerned, the orbital energies of the weakly interacting fragments in the DAN-ANT model, prior to ionization, are very close to those in the precursor molecules. The orbital energies before and after ionization therefore suggest that a larger magnitude SHI may be obtained by replacing the anthracene precursor with one whose HOMO is somewhat higher in energy. PPTA appeared to be a good choice among the set of fragments considered in the present study. Its $\mathrm{HOMO}$ was calculated to be $0.4 \mathrm{eV}$ higher in energy than that of anthracene. Assuming similar interactions between the orbitals centered on the two fragments before and after ionization, we expected SHI in the radical cation of the combined system, with a HOMO-SOMO energy gap of about $0.5 \mathrm{eV}$, i.e., $0.4 \mathrm{eV}$ larger than that of the $\mathrm{DAN}^{-\mathrm{ANT}^{\bullet+}}{ }^{-}$model. This is indeed what the calculations produced (Figure $\mathbf{S 1 2}$ ), with the SOMO centered on the PPTA fragmentclearly corresponding to the HOMO of the PPTA precursor-and energetically below three pairs of spin MOs centered on the DAN fragment. Thus, SHI may manifest itself here via a SOMO that is energetically below not just one, but several pairs of matching $\alpha$ and $\beta$ spin MOs. We also encounter such scenarios later with a different set of molecular systems.

Another pair of test system was constructed from naphthalene and triarylamine with methoxy or chlorine substituents (TAA-MeO, TAA-Cl). See Figures S13 and S14. The TAA derivatives

were chosen because they were used in related prior experimental and computational work! $16,60,61$ The HOMO of naphthalene was calculated at $-6.3 \mathrm{eV}$, while the HOMO of TAA-MeO and TAA$\mathrm{Cl}$ was at -4.8 and $-5.7 \mathrm{eV}$, respectively. The combined fragment systems both show significant $\pi$ conjugation between the fragments, and the $\alpha$ spin orbitals undergo significant relaxation upon ionization, such that an identification of SHI is less straightforward. Based on inspection of the radical ion's $\beta$ spin frontier orbitals, which more closely relate to the precursor orbitals, the systems likely fall in the SHI category.

\subsection{SHI radicals with spatially overlapping HOMO and HOMO-1 in the closed-shell parent}

Closed-shell systems with spatially disjoint occupied frontier MOs are not the only cases that will form SHI radicals upon ionization. Consider, for example, the open-shell aza-thia[7]helicene $\left(\mathrm{ATH}^{\circ}{ }^{0}\right)$ shown in Figure 1. This radical was confirmed to exhibit SHI in the publication first reporting its synthesis and characterization. ${ }^{15}$ The same study also provided experimental data for the closed-shell parent system, the ATH anion. Our computational results are collected in Figure 5 and Tables 4 and 5. As for the anions of TTM and PTM, the ATH anion's HOMO energy, $-1.8 \mathrm{eV}$ 


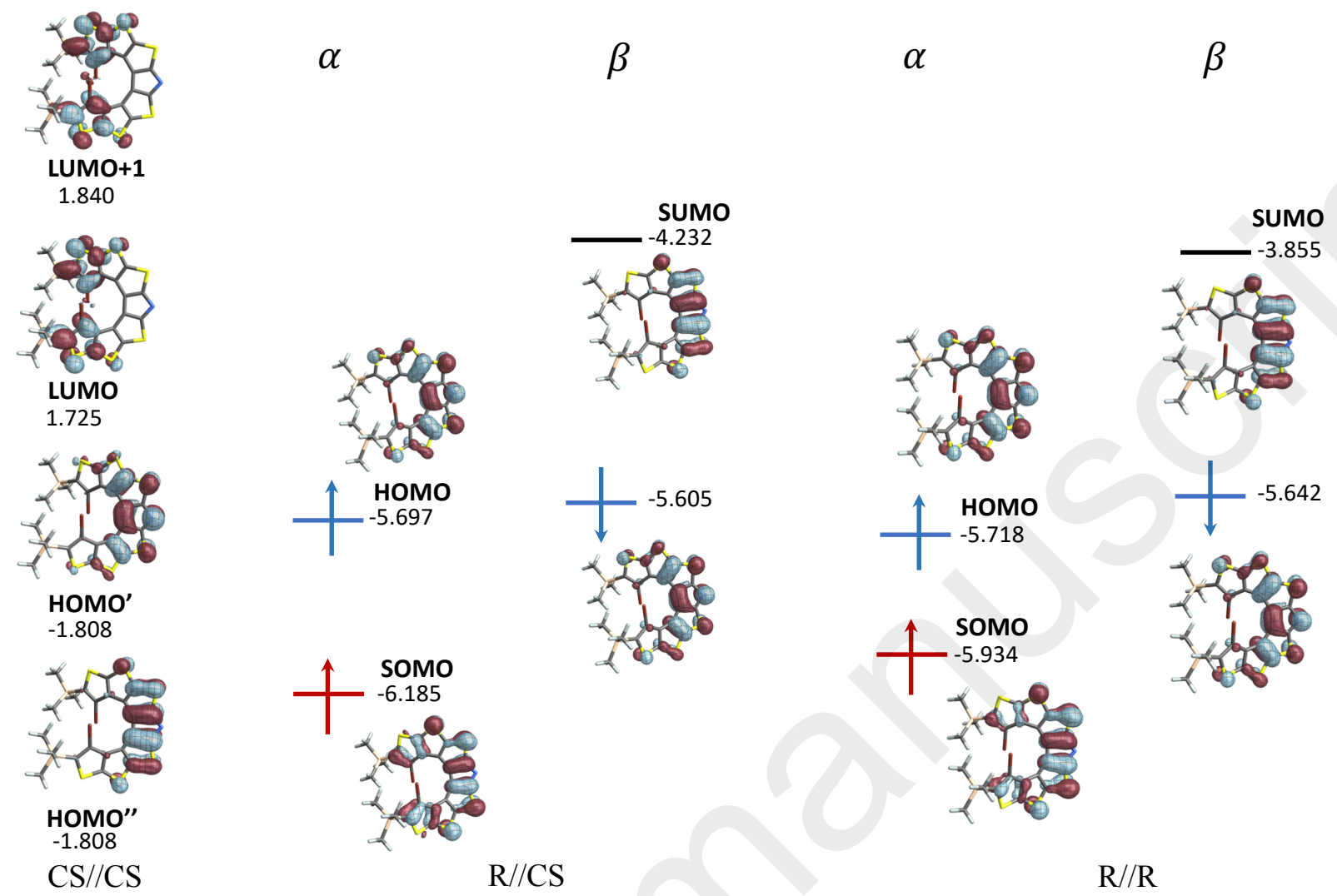

Figure 5: Orbital energies (in eV) and isosurfaces ( \pm 0.030 a.u.) of selected MOs of the ATH system. See Section 3.1 for a description of the labeling.

indicates that it is stable but not very difficult to oxidize by chemical means. What sets the ATH anion apart from the other closed-shell systems analyzed so far is that the HOMO and HOMO-1 are quasi-degenerate [less than $10^{-3} \mathrm{eV}$ difference in the MO energies with PBE0, and as little as 0.008 and $0.010 \mathrm{eV}$ difference with PBE and B3LYP respectively]. For brevity, we label the pair of doubly occupied MOs as $\mathrm{H}^{\prime}$ and $\mathrm{H}^{\prime \prime}$. It is ultimately $\mathrm{H}^{\prime \prime}$ that converts to the SOMO, upon noticeable relaxation, when the electron is removed from the anion.

There are large self-Coulomb ERIs, 5.7 and $6.5 \mathrm{eV}$, representing the repulsion between the $\alpha$ and $\beta$ spin components of $\mathrm{H}^{\prime}$ and $\mathrm{H}^{\prime \prime}$, respectively. Furthermore, because the two orbitals overlap strongly, there is a large Coulomb ERI $(5.5 \mathrm{eV})$ between them, in addition to a sizable exchange ERI. In the conceptual breakdown of the radical formation in Table 4, the loss of the self-Coulomb ERI leads - as expected - to a strong energetic stabilization of the SOMO when the system is ionized. As for the other analyzed cases, the changes in the MO energies prior to orbital relaxation correspond very closely to the values of the relevant Coulomb ERIs listed in Table 5, while XC contributions are negligible. The pathway where the occupation is removed from the $\beta$ spin component of $\mathrm{H}^{\prime \prime}$ is the most intuitive. In the initial CS//CS $\rightarrow \tilde{\mathrm{R}} / / \mathrm{CR}$ step, the remaining $\alpha$ spin 
component, the SOMO, drops $6.5 \mathrm{eV}$ in energy, while $\mathrm{H}^{\prime}$ drops by $5.5 \mathrm{eV}$ corresponding to the Coulomb ERI between $\mathrm{H}^{\prime}$ and $\mathrm{H}^{\prime \prime}$. At this point, the $\mathrm{SHI}$ is established, although subsequent orbital and structural relaxation reduce the energy gap between SOMO and HOMO significantly. The driver for the SHI is seen to be the loss of the large self-Coulomb repulsion of $\mathrm{H}^{\prime \prime}$, aided by the fact that $\mathrm{H}^{\prime}$ and $H^{\prime \prime}$ are degenerate. Likely, if $\mathrm{H}^{\prime}$ had been energetically below $\mathrm{H}^{\prime \prime}$ in the CS parent, the SHI would have been smaller in magnitude or disappeared altogether.

Rajca et al. also reported the aza-thia[7]helicene with the N-H moiety, ${ }^{15}$ labeled here as the $\mathrm{N}$-protonated form of ATH (Figure 6a). This case is similar to $\mathrm{ATH}^{\circ}{ }^{0}$ system, because the HOMO and HOMO-1 of the neutral closed-shell parent system are close in energy and the corresponding radical cation shows SHI (Figure 6). Interestingly, among the two occupied frontier MOs of the

Table 4: MO energy contributions (in eV) for the ATH system. ${ }^{(a)}$ The numbers are for the $\alpha$ spin orbitals. See also Figure 5 .

\begin{tabular}{lrrrrrrrr}
\hline $\mathrm{HOMO}^{\prime} \rightarrow$ HOMO & $\mathrm{CS} / / \mathrm{CS}$ & $\stackrel{\Delta}{\rightarrow}$ & $\tilde{\mathrm{R}} / / \mathrm{CS}$ & $\stackrel{\Delta}{\rightarrow}$ & $\mathrm{R} / / \mathrm{CS}$ & $\stackrel{\Delta}{\rightarrow}$ & $\mathrm{R} / / \mathrm{R}$ \\
\hline$T$ & & 43.68 & 0.00 & 43.68 & 1.84 & 45.52 & 0.02 & 45.54 \\
$V^{\text {nuc }}$ & $-1320^{(b)}$ & 38.88 & 0.00 & 38.88 & -2.58 & 36.30 & -5.17 & 31.12 \\
$V^{C}$ & $+1320^{(b)}$ & -68.50 & -5.54 & -74.04 & 2.37 & -71.67 & 5.12 & -66.55 \\
$V^{\mathrm{XC}}$ & & -15.86 & 0.01 & -15.85 & 0.01 & -15.85 & 0.01 & -15.83 \\
total $\varepsilon$ & -1.81 & -5.53 & -7.34 & 1.64 & -5.70 & -0.02 & -5.72 \\
& & & & & & & \\
\hline \multirow{2}{*}{$\mathrm{HOMO}^{\prime \prime} \rightarrow$ SOMO } & $\mathrm{CS} / / \mathrm{CS}$ & $\stackrel{\Delta}{\rightarrow}$ & $\tilde{\mathrm{R}} / / \mathrm{CS}$ & $\stackrel{\Delta}{\rightarrow}$ & $\mathrm{R} / / \mathrm{CS}$ & $\stackrel{\Delta}{\rightarrow}$ & $\mathrm{R} / / \mathrm{R}$ \\
\hline$T$ & 40.41 & 0.00 & 40.41 & 5.59 & 46.00 & -0.33 & 45.67 \\
$V^{\text {nuc }}$ & $-1320^{(b)}$ & 44.36 & 0.00 & -44.36 & -7.48 & 36.88 & 13.01 & 49.89 \\
$V^{C}$ & $+1320^{(b)}$ & -71.28 & -6.49 & -77.78 & 4.39 & -73.39 & -12.44 & -85.83 \\
$V^{\mathrm{XC}}$ & & -15.29 & 0.03 & -15.27 & -0.41 & -15.67 & 0.01 & -15.67 \\
total $\varepsilon$ & -1.81 & -6.47 & -8.27 & 2.09 & -6.18 & 0.25 & -5.93 \\
\hline
\end{tabular}

(a) CS: Closed-shell system. R̃: Radical without SCF, using the MOs of the closed-shell system. R: Radical system. ${ }^{(b)}$ The listed number was subtracted from $V^{\text {nuc }} / V^{C}$ to avoid large values in the other Table columns. Add it to get the full value of $V^{\text {nuc }} / V^{C}$.

Table 5: Selected Coulomb and exchange electron repulsion integrals (ERIs, in eV) for the closedshell parent system of $\mathrm{ATH}^{\bullet}$.

\begin{tabular}{|c|c|}
\hline ERI Type & ATH \\
\hline self-Coulomb ${ }^{(a)} \mathrm{HOMO}^{\prime \prime}$ & 6.494 \\
\hline Coulomb HOMO" / HOMO' & 5.543 \\
\hline self-Coulomb ${ }^{(a)} \mathrm{HOMO}^{\prime}$ & 5.715 \\
\hline exchange $\mathrm{HOMO}^{\prime \prime}$ / HOMO' & 0.442 \\
\hline
\end{tabular}

(a) This corresponds to the Coulomb repulsion between the $\alpha$ spin and the $\beta$ spin component of the MO. 
a)

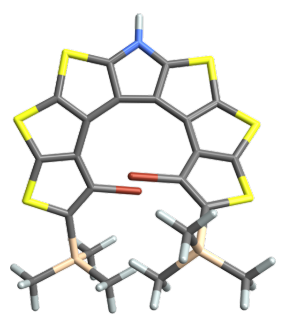

b)

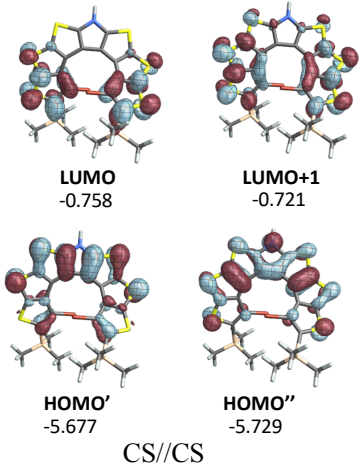

c)

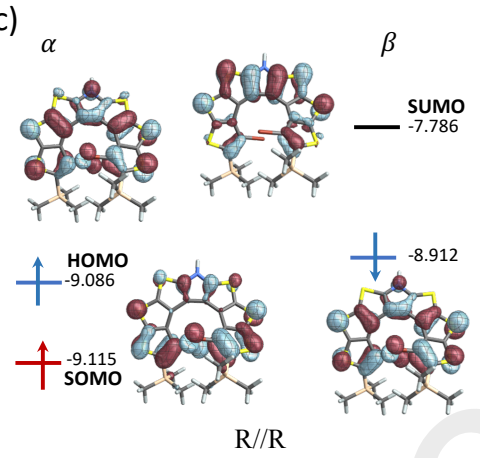

Figure 6: a) DFT-optimized structure of the N-protonated form of ATH. b) and c) Orbital energies (in $\mathrm{eV}$ ) and isosurfaces $( \pm 0.030$ a.u.) of selected MOs for the neutral closed shell parent and the corresponding radical cation for the $\mathrm{N}$-protonated form of ATH, respectively.

closed shell parent, $\mathrm{HOMO}^{\prime \prime}$ has a node at $\mathrm{N}$, whereas $\mathrm{HOMO}^{\prime}$ does not. Consequently, protonation at $\mathrm{N}$ stabilizes $\mathrm{HOMO}^{\prime}$ more strongly, breaking the degeneracy. Per our previous analysis of $\mathrm{ATH}^{\circ}$, this should lead to a smaller-magnitude SHI, and this is indeed the case (see Figure 6r). We note that the $\beta$ spin SUMO is clearly reflected in the spin density. The density difference $\Delta \rho$ between the closed-shell parent and the radical cation (using the radical geometry) reflects some of the features of the $\alpha$ spin SOMO, but also evidences the orbital relaxation between CS and the radical. See Figures $\mathrm{S16} a$ and $\mathrm{S16} b$, respectively.

These results hint at a design strategy for SHI systems with strongly overlapping HOMO and HOMO-1 orbitals: If the two orbitals are nearly degenerate, but if the self-Coulomb ERI for one of them is considerably larger than the self-Coulomb ERIs for the other orbitals and the ERIs between them (with differences between 0.8 and $1.0 \mathrm{eV}$, in the case of ATH), there may be enough of a difference in the energy changes of the two orbitals upon ionization such that the SHI system emerges as the ground state of the radical. In this case, as for the other SHI systems, the loss of a particularly strong self-Coulomb repulsion upon formation of the radical must also be considered as the determining factor in the total energy that renders the SHI electronic structure of the radical more stable than the alternatives.

The case of the neutral $\mathrm{ETBN}^{\bullet 0}$ radical (Figure 1), which was previously synthesized and

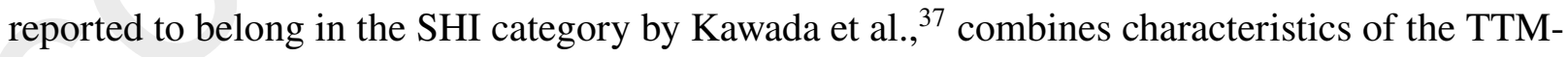
$\mathrm{PPTA}^{\circ}$ and $\mathrm{ATH}^{\circ}{ }^{0}$ systems. Due to space considerations, the data are presented in Figure $\mathrm{S} 17$ and Tables S5 and S6 in the SI. In the closed-shell parent, HOMO and HOMO-1 are spatially close, with a strong Coulomb repulsion of $7.7 \mathrm{eV}$ between them, but the HOMO self-repulsion is much larger $(9.2 \mathrm{eV})$, a situation that is qualitatively similar to ATH. It is HOMO-4 of the closed-shell parent of $\mathrm{ETBN}^{\bullet}$ that ultimately becomes the SOMO, for the reason that it is spatially disjoint from HOMO and HOMO-1 and has a much smaller Coulomb repulsion with them $(2.9 \mathrm{eV}$ for 
the Coulomb ERI between HOMO-4 and HOMO). Therefore, similar to the TTM-PPTA system, the energy of this orbital drops much less upon ionization, such that the CS HOMO-4 becomes the radical HOMO. In the R//CS system, the SOMO is below both HOMO and HOMO-1, with the HOMO-1 - SOMO inversion being ATH-like, and the HOMO - SOMO inversion being TTMPPTA-like. However, the former SHI mechanism does not persist when the radical's structure relaxes, going from $\mathrm{R} / / \mathrm{CS}$ to $\mathrm{R} / / \mathrm{R}$, whereas the latter does. In $\mathrm{R} / / \mathrm{R}$, there is a very large gap in the energies of the SOMO and the SUMO, which is principally caused by the strong Coulomb ERI between these two very compact orbitals: The SUMO energy contains the repulsion, but the SOMO energy does not.

A somewhat different scenario is represented by the chiral bicarbazole derivative $\left(\mathrm{BCz}^{\bullet+}\right)$ of Figure 1, studied previously in Reference 17. The data are collected in Figure S19 and Tables S7 and S8. The CS parent system represents two linked equivalent chromophores. In the weakly interacting dimer, the HOMO and HOMO-1 are therefore out-of-phase and in-phase linear combinations of equivalent highest occupied fragment orbitals, and nearly degenerate. Unlike the ATH system examined previously, the Coulomb ERIs within and between HOMO and HOMO-1 are nearly the same. Starting with CS//CS, vertical ionization does not yet produce an SHI system in the R//CS stage in the same sense as the other SHI cases studied herein, because SOMO is the highest among the $\alpha$ spin orbitals. There is, however, a very strong mismatch in energy between the $\alpha$ and $\beta$ spin component of the HOMO in $\mathrm{R} / / \mathrm{CS}$, such that the $\beta$ component is above the $\alpha$ spin SOMO. SHI within the set of $\alpha$ spin orbitals emerges when the radical structure relaxes to R//R. During this step, the $\alpha$ spin HOMO and $\beta$ spin SUMO become localized on different carbazole fragments. The SOMO is not as cleanly identifiable as in some of the other cases studied, which was mentioned earlier in Section 3.2. The calculated spin density (Figure S20) corresponds clearly to the $\beta$ spin electron hole represented by the SUMO. The $\alpha$ spin counterpart of the SUMO in fact corresponds to a 50-50 linear combination of the second and third highest occupied $\alpha$ spin MO (see Figure S21). Both these MOs are more than $0.5 \mathrm{eV}$ below the pair of orbitals constituting the HOMO, and therefore the SHI assignment of the radical is unambiguous. These results hint at another possibility for SHI to occur, namely if the orbitals of a closed-shell dimer of weakly to moderately strongly interacting identical fragments localize upon adiabatic ionization. The end result is then not unlike the end result of the SHI design strategy stated in Section 3.3 , in the sense that in the final step the HOMO and SOMO in the radical become spatially disjoint.

\subsection{Further Analyses}

To complete our exploration of SHI candidates and further test the design strategies developed thus far, we considered linkages between one of three naphthalene diimides axially substituted 


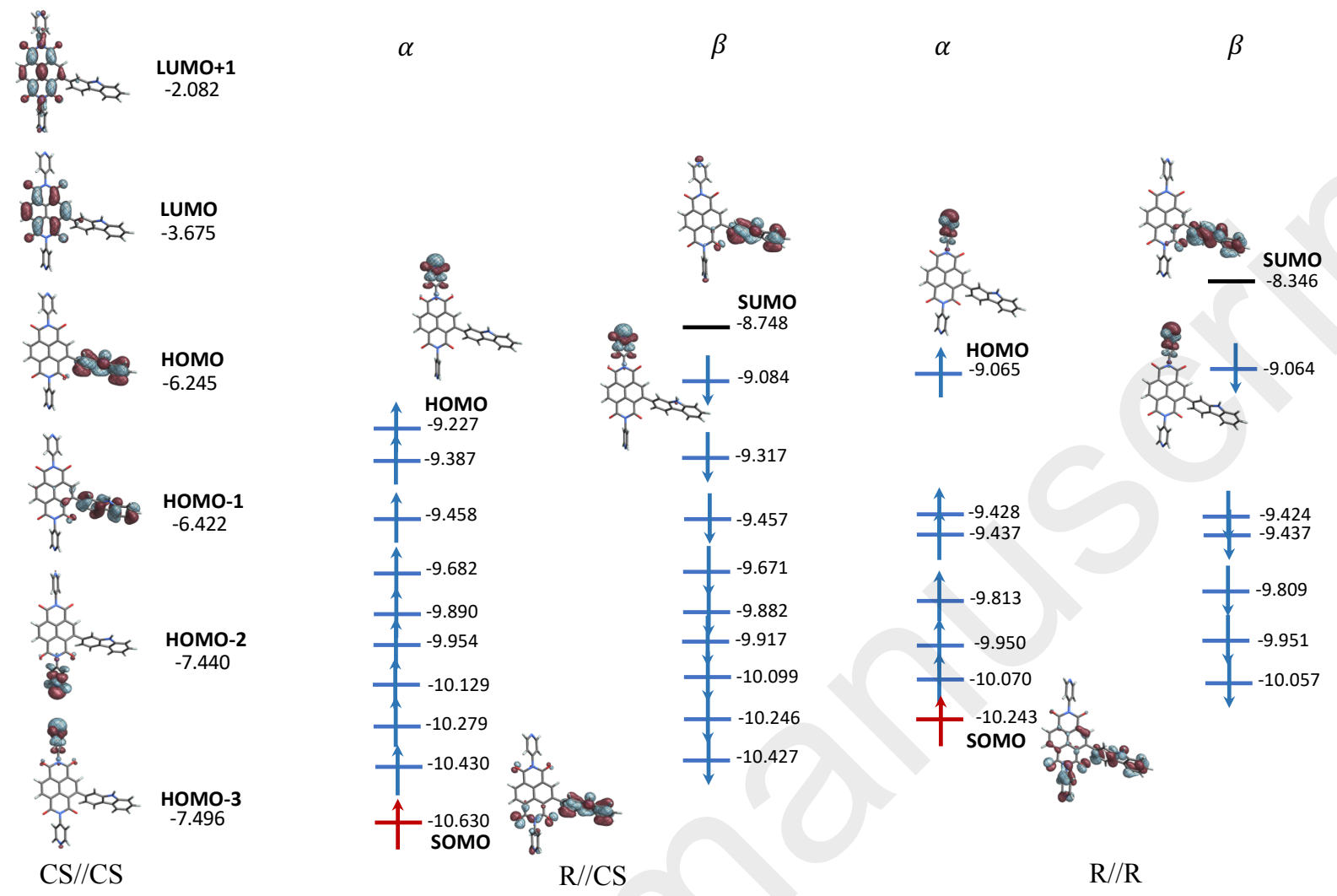

Figure 7: Orbital energies (in eV) and isosurfaces $( \pm 0.030$ a.u.) of selected MOs of the NDI-Mol2 system. See Section 3.1 for a description of the labeling.

a)

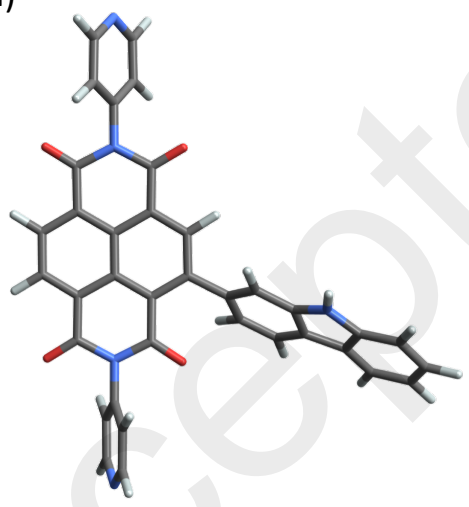

b)

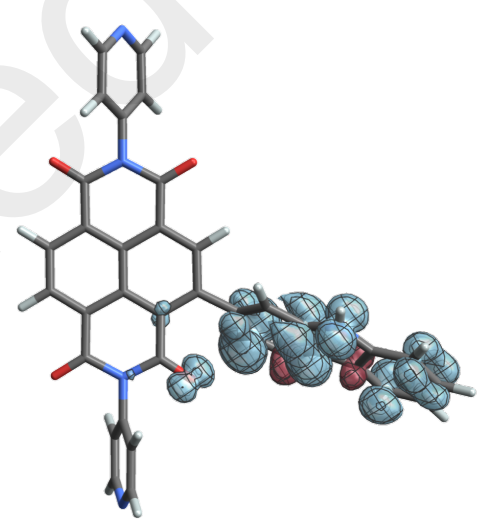

c)

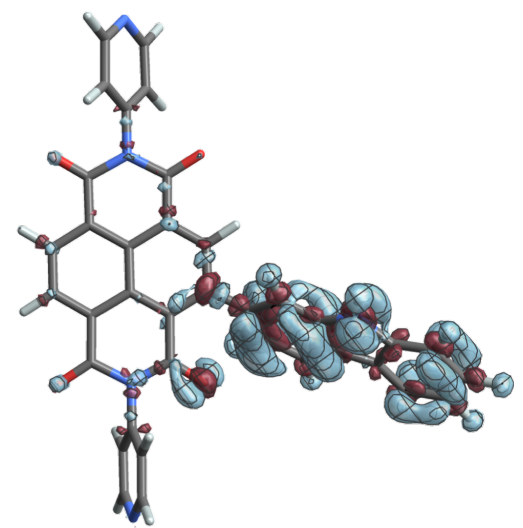

Figure 8: a) DFT-optimized structure of NDI-Mol-2 ${ }^{\bullet+}$. b) Isosurface $( \pm 0.002$ a.u. $)$ of the spin density of NDI-Mol-2 ${ }^{\bullet}$. c) Isosurface $( \pm 0.002$ a.u. $)$ of the density difference between the neutral and the radical cation of NDI-Mol-2 system.

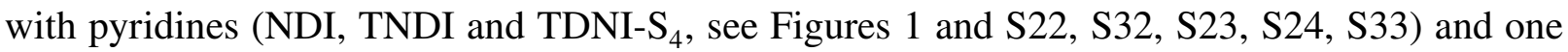
of fifteen organic molecule precursors (Mol-X, with $X=1-15$, see Figures 1 and $[25)$. Naph- 
thalene diimides have come under intense scrutiny because of their electron-deficient character, which makes them very promising electronic materials $32+34,62$ One naphthalene diimide derivative was selected because of its electron-poor properties, as already reported in previous studies. $\frac{34,63}{6}$ The thionated NDIs ${ }^{\sqrt[35]{36}}$ look to be valuable promising towards generating high electron deficient molecules,, 34 therefore, the thionated analogue of NDI was also studied. The thionated $\mathrm{TDNI}_{4}$ derivate was chosen because it seems easier to synthesize than TDNI. The functionalization of the NDIs via core substitution has attracted high interest in organic electronics, as it produces modifications in the optical and electronic properties $\frac{3264}{68}$ In this work, electron-rich organic molecules (see Mol-X in Figure $\mathrm{S} 25$ for the full set) were selected to be linked to these

Table 6: MO energy contributions (in eV) for the NDI-Mol-2 system. ${ }^{(a)}$ The numbers are for the $\alpha$ spin orbitals. See also Figure 7 .

\begin{tabular}{lrrrrrrrr}
\hline HOMO $\rightarrow$ SOMO & CS // CS & $\stackrel{\Delta}{\rightarrow}$ & $\tilde{\mathrm{R}} / / \mathrm{CS}$ & $\stackrel{\Delta}{\rightarrow}$ & $\mathrm{R} / / \mathrm{CS}$ & $\stackrel{\Delta}{\rightarrow}$ & $\mathrm{R} / / \mathrm{R}$ \\
\hline$T$ & & 38.07 & 0.00 & 38.07 & 9.11 & 47.18 & -2.11 & 45.08 \\
$V^{\text {nuc }}$ & $-1000^{(b)}$ & 57.51 & 0.00 & 57.51 & -52.56 & 4.95 & -26.96 & -22.01 \\
$V^{C}$ & $+1000^{(b)}$ & -86.17 & -6.22 & -92.39 & 47.36 & -45.03 & 28.87 & -16.16 \\
$V^{\mathrm{XC}}$ & & -15.65 & 0.03 & -15.62 & -2.11 & -17.73 & 0.58 & -17.15 \\
total $\varepsilon$ & -6.24 & -6.19 & -12.44 & 1.81 & -10.63 & 0.39 & -10.24 \\
& & & & & & & \\
\hline \multirow{2}{*}{$\mathrm{HOMO}-3 \rightarrow \mathrm{HOMO}$} & $\mathrm{CS} / / \mathrm{CS}$ & $\stackrel{\Delta}{\rightarrow}$ & $\tilde{\mathrm{R}} / / \mathrm{CS}$ & $\stackrel{\Delta}{\rightarrow}$ & $\mathrm{R} / / \mathrm{CS}$ & $\stackrel{\Delta}{\rightarrow}$ & $\mathrm{R} / / \mathrm{R}$ \\
\hline$T$ & & 52.00 & 0.00 & 52.00 & 0.57 & 52.58 & -0.07 & 52.51 \\
$V^{\text {nuc }}$ & $-870^{(c)}$ & 32.53 & 0.00 & 32.53 & 7.19 & 39.72 & 3.24 & 42.96 \\
$V^{C}$ & $+870^{(c)}$ & -72.99 & -1.24 & -74.22 & -8.13 & -82.36 & -3.04 & -85.39 \\
$V^{\mathrm{XC}}$ & & -19.05 & 0.00 & -19.05 & -0.12 & -19.17 & 0.03 & -19.14 \\
total $\varepsilon$ & -7.50 & -1.24 & -8.73 & -0.50 & -9.23 & 0.16 & -9.06 \\
\hline
\end{tabular}

(a) CS: Closed-shell system. R̃: Radical without SCF, using the MOs of the closed-shell system. R:

Radical system. ${ }^{(b),(c)}$ The listed number was subtracted from $V^{\text {nuc }} / V^{C}$ to avoid large values in the other Table columns. Add it to get the full value of $V^{\text {nuc }} / V^{C}$.

Table 7: Selected Coulomb and exchange electron repulsion integrals (ERIs, in eV) for the closedshell parent systems of NDI-Mol-2 ${ }^{\bullet+}$ and TNDI-Mol-3 ${ }^{\bullet+}$.

\begin{tabular}{|c|c|c|}
\hline ERI Type & NDI-Mol-2 & TNDI-Mol-3 \\
\hline self-Coulomb ${ }^{(a)}$ HOMO-3 & 12.949 & 5.609 \\
\hline self-Coulomb ${ }^{(a)}$ HOMO-1 & 5.725 & 5.281 \\
\hline Coulomb HOMO-3 / HOMO & 1.235 & 1.976 \\
\hline Coulomb HOMO-1 / HOMO & 5.274 & 4.638 \\
\hline self-Coulomb ${ }^{(a)}$ HOMO & 6.222 & 5.111 \\
\hline exchange HOMO-1 / HOMO & 0.305 & 0.583 \\
\hline
\end{tabular}

(a) This corresponds to the Coulomb repulsion between the $\alpha$ spin and the $\beta$ spin component of the MO. 
NDIs. Mol-X has HOMO energies in the range of -5.0 to $-6.0 \mathrm{eV}$, while the naphthalene diimides have HOMO energies of -7.6 (NDI), $-6.6 \mathrm{eV}$ (TNDI) and $-6.6 \mathrm{eV}\left(\mathrm{TDNI}_{-} \mathrm{S}_{4}\right.$ ), such that a wide range of orbital energy differences between the fragments could be studied in the context of SHI for the resulting radical cations. Figures S26, S27 and S34 display the optimized structures of the radical cations, along with the corresponding HOMO-SOMO energy differences. All of the systems exhibit SHI to some degree, as indicated by the positive values listed for the energy gaps. Among them, NDI-Mol-2 ${ }^{+}(1.18 \mathrm{eV}$ gap $)$ and TNDI-Mol-3 ${ }^{\bullet+}(0.97 \mathrm{eV}$ gap $)$ gave particularly large energy differences between the SOMO and the highest occupied MO of the same spin and were selected for a more detailed analysis. The relevant MO isosurface plots and numerical data can be found in Tables 6, 7, and S9, and in Figures 7 and $\mathbf{S 2 8}$.

Inspection of the MOs for both systems shows a clear correspondence between the HOMO of the CS parent and the SOMO and SUMO in the radical ions. Furthermore, in both systems the SOMO is below a range of other orbitals that are clearly identifiable as matching $\alpha$ and $\beta$ spin pairs. Also, in both systems, the calculated spin density reflects the $\beta$ spin electron hole of the SUMO (Figures $8 \mathrm{~b}$ and $\mathrm{S} 29 \mathrm{~b}$ ), and the density difference $\Delta \rho$ between the closed-shell parent and the radical using the radical geometries is representative of the HOMO of the closed-shell system, save for the orbital relaxation (Figures $8 \mathrm{c}$ and $\mathrm{S} 29 \mathrm{k}$ ). In the closed shell parent systems, HOMO and HOMO-1 are Mol-X centered. Further below in energy are the naphthalene diimidecentered orbitals and other orbitals centered on the Mol-X fragment. In the radicals, the SOMO is localized on the Mol-X fragment, whereas the HOMO is centered on the naphthalene diimide fragment. As in the case of TTM-PPTA, there is a large Coulomb repulsion between the $\alpha$ and $\beta$ spin component of the CS HOMO (6.2 eV for NDI-Mol-2, and $5.1 \mathrm{eV}$ for TNDI-Mol-3, see Table 7 for the ERI values). The initial drop in energy of this orbital, going from CS//CS to $\tilde{\mathrm{R}} / / \mathrm{CS}$, essentially corresponds to the loss of this ERI in the energy HOMO $\rightarrow$ SOMO, and, again like for TTM-PPTA, there is an increase of the MO energy when the orbitals relax, followed by substantial but mostly canceling changes predominantly in $V^{C}$ and $V^{\text {nuc }}$ as the radical changes into its equilibrium structure. The analysis of the energy contributions to the orbital that becomes the HOMO in the radical (HOMO $\rightarrow$ SOMO) is also similar to what we found for TTM-PPTA, and, likewise, very similar energetic changes appear in the isolated moiety that is being ionized (Mol$X$, see Figures S36 and S37]. Because the orbitals centered on the naphthalene diimide and Mol$\mathrm{X}$ fragments do not overlap strongly, the orbitals centered on the naphthalene diimide fragment drop in energy only modestly when the system gets ionized. There is some orbital relaxation taking place upon ionization, but as already pointed out the CS HOMO and the radical SOMO and SUMO isosurface plots appear virtually identical, such that SHI can be assigned for the radicals straightforwardly. The SHI appears for the same reasons as discussed in Sections 3.2 and 3.3 .

The linkage between NDI and Mol-12 shows the largest HOMO-SOMO energy difference 
$(1.33 \mathrm{eV})$, followed by NDI-Mol-2 ${ }^{\bullet+}(1.18 \mathrm{eV})$. Both NDI systems have the same carbazole unit in their core. The difference is a $t$-Bu group added in position 6 of the carbazole of NDI-Mol-12 (see Figure $\mathrm{S} 23$. This increases the stability of the radical carbazole cation, and also causes the $\mathrm{SHI}$ gap to be $0.15 \mathrm{eV}$ larger than that for $\mathrm{R}=\mathrm{H}$. We expect the $t$-Bu group to lend the radical additional chemical stability. Analyzing the CS NDI-Mol-12, the HOMO level is $-6.13 \mathrm{eV}$, which is very similar in energy than that for NDI-Mol-2 (-6.25 eV) (Figure S30). The self-Coulomb ERIs for the CS HOMO of both systems are also very similar (Table[S10).

A final test was carried out, with chlorine substituting hydrogens of the pyridine groups of TNDI, to stabilize this fragment's HOMO energetically via inductive effects. The results of this calculation for the radical ion are displayed in Figure $\mathrm{S} 38$, The system also presents a clear-cut SHI case, but it has a smaller SOMO-HOMO energy difference than TNDI-Mol-3 ${ }^{\bullet+}(0.57 \mathrm{eV}$ vs. 0.97 $\mathrm{eV})$. The smaller energy gap SHI is essentially inherited from the lowering of the HOMO level of TNDI upon substitution, which brings the HOMO of the radical closer to the SOMO. In comparison, the SOMOs in the two systems have nearly identical energies. This calculation shows that in the computational design of SHI systems composed of electronically relatively weakly interacting fragments, the orbital energies of the precursor fragments and the Coulomb ERIs involving the HOMO of the closed-shell parent system of the radical will to a large extent determine the size of the SOMO-HOMO energy gap.

\section{Conclusions}

The present study has focused on conditions that would favor a radical where the SOMO, the un-paired molecular orbital associated with the radical character of the system, lies energetically below one or several paired molecular orbitals. Given that the highest occupied / unoccupied MO level is associated with electron detachment / attachment, a growing body of literature is focusing on the peculiar chemical and physical properties of such SHI radicals. From a practical theoretical perspective, the frontier MOs from KS DFT calculations with approximate functionals have long been known to provide semi-quantitative, if not quantitative, data for ionization processes, along with chemically intuitive interpretations. We therefore studied SHI in radicals by such calculations.

The analysis provided a rather consistent picture: When the radical is conceptually created from a parent system with a closed-shell electron configuration and one more electron, the electrostatic repulsion among the frontier orbitals, and the repulsion between the $\alpha$ and $\beta$ spin component of a given closed-shell MO (dubbed the 'self-Coulomb' repulsion of the MO in Section 3 ) are key to understanding the occurrence of SHI in the radical. In most of the reported SHI cases, the SOMO and HOMO are spatially disjoint and not degenerate. In one type of scenario, SHI may 
occur if the HOMO of the CS parent has a large self-Coulomb repulsion, such that its energy drops by a lot upon ionization. Not too strong spatial overlap between this orbital and the CS HOMO-1 means the Coulomb repulsion between the two orbitals is comparatively weak, and consequently the latter MO drops far less in energy upon ionization. If the HOMO-1 of the closed-shell system is not too low in energy, this may result in SHI. Here, and in the other cases, the initial loss of a large self-Coulomb ERI must be considered as the main factor that causes the total energy of the SHI radical to be lower than the total energies of alternative electronic configurations.

In another scenario, the system is composed of two equivalent fragments, and the highest occupied orbitals of the two fragments interact more or less strongly to form in-phase and out-ofphase linear combinations constituting the CS HOMO and HOMO-1. Upon ionization, SHI may occur if the SOMO and HOMO of the radical localize on separate fragments, thus establishing a situation with spatially disjoint orbitals not unlike the end result in the first scenario. A third scenario is represented by the studied aza-thia[7]helicene. Here, the HOMO and HOMO-1 of the CS parent are effectively degenerate, and they both have large but still very different selfCoulomb repulsion. Although the two MOs overlap spatially, which tends to cause significant orbital relaxation upon ionization, the loss of the larger of the two Coulomb repulsion terms in the total energy may favor the SHI ground state configuration of the radical. The present calculations also show that the SOMO-HOMO energy gap in a multi-fragment SHI radical can be tuned via optimizing the HOMO energies of the precursor molecules.

We have shown herein that considering SHI from the perspective of a closed-shell interacting parent system offers insight into the reasons why SHI radicals form in the first place. This insight then led to the formulation of relatively simple design strategies that we were able to confirm 'in silico'. A combination of these insights with information about relative frontier energies and oxidation/ionization potentials of separate fragments would seem very promising for the computational pre-screening and the computational and experimental design of new SHI systems. Ideally, this would take place in a close collaboration between synthetic and computational chemists, to predict, verify, and rationalize the interesting features of compounds with SHI, such as an increased chemical stability, which is of prime importance for potential applications in optoelectronic devices. Although it is not obvious how the electronic configuration in radicals with and without SHI precisely relates to thermodynamic stability, 19 increased photostability of SHI radicals vs. non-SHI radicals has been noted in prior research. 9 Evidently, for the rational design of SHI materials with specifically tailored properties, future theoretical research will have to tease out the exact relationships between SHI itself and the desired properties. Even so, it is clear that SHI is a desirable feature in itself, and likely a source of unusual and sought-after properties of molecular materials. This view is supported by reference 18 , which states that systems with SHI configurations (referred to there as quasi closed-shell) ' [...] are not only unusual, but also ex- 
tremely attractive as components of molecular electronic devices and various switches. Moreover, they elegantly illustrate how a purely theoretical concept — an orbital approximation — provides solid ground for their unexpected yet experimentally detected behavior.'

\section{Acknowledgments}

We acknowledge the Center for Computational Research $(\mathrm{CCR})^{69}$ at the University at Buffalo for providing computational resources. This study has been supported by grant CHE-1855470 from the National Science Foundation.

\section{Supporting Information Available}

Isosurfaces of molecular orbitals, additional analyses of the molecular orbital energy contributions, additional collections of Coulomb and exchange electron repulsion integrals, and test calculations with other functionals or with solvent effects.

\section{References}

(1) Zeng, Z.; Shi, X.; Chi, C.; López Navarrete, J. T.; Casado, J.; Wu, J. Pro-aromatic and anti-aromatic $\pi$-conjugated molecules: an irresistible wish to be diradicals. Chem. Soc. Rev. 2015, 44, 6578-6596.

(2) Abe, M. Diradicals. Chem. Rev. 2013, 113, 7011-7088.

(3) Peng, Q.; Obolda, A.; Zhang, M.; Li, F. Organic Light-Emitting Diodes Using a Neutral $\pi$ Radical as Emitter: The Emission from a Doublet. Angew. Chem. Int. Ed. 2015, 54, 70917095.

(4) Ai, X.; Evans, E. W.; Dong, S.; Gillett, A. J.; Guo, H.; Chen, Y.; Hele, T. J. H.; Friend, R. H.; Li, F. Efficient radical-based light-emitting diodes with doublet emission. Nature 2018, 563, 536-540.

(5) Ai, X.; Chen, Y.; Feng, Y.; Li, F. A Stable Room-Temperature Luminescent Biphenylmethyl Radical. Angew. Chem. Int. Ed. 2018, 57, 2869-2873.

(6) Ni, Y.; Gopalakrishna, T. Y.; Phan, H.; Kim, T.; Herng, T. S.; Han, Y.; Tao, T.; Ding, J.; Kim, D.; Wu, J. 3D global aromaticity in a fully conjugated diradicaloid cage at different oxidation states. Nat. Chem. 2020, 12, 242-248. 
(7) Sugawara, T.; Komatsu, H.; Suzuki, K. Interplay between magnetism and conductivity derived from spin-polarized donor radicals. Chem. Soc. Rev. 2011, 40, 3105-3118.

(8) Abdurahman, A.; Hele, T. J. H.; Gu, Q.; Zhang, J.; Peng, Q.; Zhang, M.; Friend, R. H.; Li, F.; Evans, E. W. Understanding the luminescent nature of organic radicals for efficient doublet emitters and pure-red light-emitting diodes. Nature Mat. 2020, 19, 1224-1229.

(9) Peng, Q.; Chen, X.-K.; Gu, Q.; Dong, S.; Guo, H.; Evans, E. W.; Gillett, A. J.; Ai, X.; Zhang, M.; Credgington, D. et al. High stability and luminescence efficiency in donoracceptor neutral radicals not following the Aufbau principle. Nature Mat. 2019, 18, 977984.

(10) Armet, O.; Veciana, J.; Rovira, C.; Riera, J.; Castaner, J.; Molins, E.; Rius, J.; Miravitlles, C.; Olivella, S.; Brichfeus, J. Inert carbon free radicals. 8. Polychlorotriphenylmethyl radicals: synthesis, structure, and spin-density distribution. J. Phys. Chem. 1987, 91, 56085616.

(11) Fox, M. A.; Gaillard, E.; Chen, C. C. Photochemistry of stable free radicals: the photolysis of perchlorotriphenylmethyl radicals. J. Am. Chem. Soc. 1987, 109, 7088-7094.

(12) Kusamoto, T.; Kume, S.; Nishihara, H. Realization of SOMO-HOMO Level Conversion for a TEMPO-Dithiolate Ligand by Coordination to Platinum(II). J. Am. Chem. Soc. 2008, $130,13844-13845$.

(13) Kobayashi, Y.; Yoshioka, M.; Saigo, K.; Hashizume, D.; Ogura, T. Hydrogen-BondingAssisted Self-Doping in Tetrathiafulvalene (TTF) Conductor. J. Am. Chem. Soc. 2009, 131, 9995-10002.

(14) Gryn'ova, G.; Marshall, D. L.; Blanksby, S. J.; Coote, M. L. Switching radical stability by pH-induced orbital conversion. Nat. Chem. 2013, 5, 474-481.

(15) Wang, Y.; Zhang, H.; Pink, M.; Olankitwanit, A.; Rajca, S.; Rajca, A. Radical Cation and Neutral Radical of Aza-thia[7]helicene with SOMO-HOMO Energy Level Inversion. J. Am. Chem. Soc. 2016, 138, 7298-7304.

(16) Cho, E.; Coropceanu, V.; Brédas, J.-L. Organic Neutral Radical Emitters: Impact of Chemical Substitution and Electronic-State Hybridization on the Luminescence Properties. J. Am. Chem. Soc. 2020, 142, 17782-17786 PMID: 32997939. 
(17) Kasemthaveechok, S.; Abella, L.; Jean, M.; Cordier, M.; Roisnel, T.; Vanthuyne, N.; Guizouarn, T.; Cador, O.; Autschbach, J.; Crassous, J.; Favereau, L. Axially and Helically Chiral Radical Bicarbazoles: SOMO-HOMO Level Inversion and Chirality Impact on the Stability of Mono- and Diradical Cations. J. Am. Chem. Soc. 2020, 142, 20409-20418.

(18) Gryn'ova, G.; Coote, M. L.; Corminboeuf, C. Theory and practice of uncommon molecular electronic configurations. WIREs Comput. Mol. Sci. 2015, 5, 440-459.

(19) Gryn'ova, G.; Coote, M. L. Origin and Scope of Long-Range Stabilizing Interactions and Associated SOMO-HOMO Conversion in Distonic Radical Anions. J. Am. Chem. Soc. 2013, $135,15392-15403$.

(20) Sakurai, H.; Izuoka, A.; Sugawara, T. Design, Preparation, and Electronic Structure of HighSpin Cation Diradicals Derived from Amine-Based Spin-Polarized Donors. J. Am. Chem. Soc. 2000, 122, 9723-9734.

(21) Izuoka, A.; Hiraishi, M.; Abe, T.; Sugawara, T.; Sato, K.; Takui, T. Spin Alignment in Singly Oxidized Spin-Polarized Diradical Donor: Thianthrene Bis(nitronyl nitroxide). J. Am. Chem. Soc. 2000, 122, 3234-3235.

(22) Nakazaki, J.; Chung, I.; Matsushita, M. M.; Sugawara, T.; Watanabe, R.; Izuoka, A.; Kawada, Y. Design and preparation of pyrrole-based spin-polarized donors. J. Mater. Chem. 2003, 13, 1011-1022.

(23) Sugawara, T.; Matsushita, M. M. Spintronics in organic $\pi$-electronic systems. J. Mater. Chem. 2009, 19, 1738-1753.

(24) Komatsu, H.; Mogi, R.; Matsushita, M. M.; Miyagi, T.; Kawada, Y.; Sugawara, T. Synthesis and properties of TSF-based spin-polarized donor. Polyhedron 2009, 28, 1996-2000 Proceedings of the 11th International Conference on Molecule-based Magnets (ICMM 2008).

(25) Nakazaki, J.; Matsushita, M. M.; Izuoka, A.; Sugawara, T. Novel spin-polarized TTF donors affording ground state triplet cation diradicals. Tet. Lett. 1999, 40, 5027-5030.

(26) Guasch, J.; Grisanti, L.; Souto, M.; Lloveras, V.; Vidal-Gancedo, J.; Ratera, I.; Painelli, A.; Rovira, C.; Veciana, J. Intra- and Intermolecular Charge Transfer in Aggregates of Tetrathiafulvalene-Triphenylmethyl Radical Derivatives in Solution. J. Am. Chem. Soc. 2013, 135, 6958-6967 PMID: 23517105. 
(27) Souto, M.; Rovira, C.; Ratera, I.; Veciana, J. TTF-PTM dyads: from switched molecular self assembly in solution to radical conductors in solid state. CrystEngComm 2017, 19, 197206.

(28) Medina Rivero, S.; Shang, R.; Hamada, H.; Yan, Q.; Tsuji, H.; Nakamura, E.; Casado, J. Non-Aufbau Spiro-Conjugated Quinoidal \& Aromatic Charged Radicals. Bull. Chem. Soc. Jpn. 2021, 94, 989-996.

(29) Kumar, A.; Sevilla, M. D. SOMO-HOMO Level Inversion in Biologically Important Radicals. J. Phys. Chem. B 2018, 122, 98-105 PMID: 29240424.

(30) Franchi, P.; Mezzina, E.; Lucarini, M. SOMO-HOMO Conversion in Distonic Radical Anions: An Experimental Test in Solution by EPR Radical Equilibration Technique. J. Am. Chem. Soc. 2014, 136, 1250-1252 PMID: 24410365.

(31) Glaesemann, K. R.; Schmidt, M. W. On the Ordering of Orbital Energies in High-Spin ROHF. J. Phys. Chem. A 2010, 114, 8772-8777.

(32) Suraru, S.-L.; Würthner, F. Strategies for the Synthesis of Functional Naphthalene Diimides. Angew. Chem. Int. Ed. 2014, 53, 7428-7448.

(33) Al Kobaisi, M.; Bhosale, S. V.; Latham, K.; Raynor, A. M.; Bhosale, S. V. Functional Naphthalene Diimides: Synthesis, Properties, and Applications. Chem. Rev. 2016, 116, 1168511796 PMID: 27564253.

(34) Kumar, S.; Shukla, J.; Kumar, Y.; Mukhopadhyay, P. Electron-poor arylenediimides. Org. Chem. Front. 2018, 5, 2254-2276.

(35) Kozycz, L. M.; Guo, C.; Manion, J. G.; Tilley, A. J.; Lough, A. J.; Li, Y.; Seferos, D. S. Enhanced electron mobility in crystalline thionated naphthalene diimides. J. Mater. Chem. C 2015, 3, 11505-11515.

(36) Pearce, N.; Davies, E. S.; Horvath, R.; Pfeiffer, C. R.; Sun, X.-Z.; Lewis, W.; McMaster, J.; George, M. W.; Champness, N. R. Thionated naphthalene diimides: tuneable chromophores for applications in photoactive dyads. Phys. Chem. Chem. Phys. 2018, 20, 752-764.

(37) Nakazaki, J.; Ishikawa, Y.; Izuoka, A.; Sugawara, T.; Kawada, Y. Preparation of isolable ion-radical salt derived from TTF-based spin-polarized donor. Chem. Phys. Lett. 2000, 319, 385-390. 
(38) Frisch, M. J.; Trucks, G. W.; Schlegel, H. B.; Scuseria, G. E.; Robb, M. A.; Cheeseman, J. R.; Scalmani, G.; Barone, V.; Petersson, G. A.; Nakatsuji, H. et al. "Gaussian 16 Revision C.01”, Gaussian, Inc., Wallingford CT, 2016. URL: www.gaussian.com.

(39) Adamo, C.; Barone, V. Toward reliable density functional methods without adjustable parameters: The PBE0 model. J. Chem. Phys. 1999, 110, 6158-6170.

(40) Weigend, F.; Ahlrichs, R. Balanced basis sets of split valence, triple zeta valence and quadruple zeta valence quality for $\mathrm{H}$ to Rn: Design and assessment of accuracy. Phys. Chem. Chem. Phys. 2005, 7, 3297-3305.

(41) Weigend, F. Accurate Coulomb-fitting basis sets for H to Rn. Phys. Chem. Chem. Phys. 2006, 8, 1057-1065.

(42) Grimme, S.; Antony, J.; Ehrlich, S.; Krieg, H. A consistent and accurate ab initio parametrization of density functional dispersion correction (DFT-D) for the 94 elements H-Pu. J. Chem. Phys. 2010, 132, 154104.

(43) Scalmani, G.; Frisch, M. J. Continuous surface charge polarizable continuum models of solvation. I. General formalism. J. Chem. Phys. 2010, 132, 114110.

(44) Tomasi, J.; Mennucci, B.; Cammi, R. Quantum mechanical continuum solvation models. Chem. Rev. 2005, 105, 2999-3093.

(45) Aprà, E. et al. NWChem: Past, present, and future. J. Chem. Phys. 2020, 152, 184102.

(46) NWChem quantum chemistry program package. URL http: / /wWw . nwchem-sw . org and https: / / nwchemgit.github.io/. Accessed 01/21.

(47) URL https://github.com/jautschbach (accessed 02/21).

(48) Klamt, A.; Schüürmann, G. COSMO: A new approach to dielectric screening in solvents with explicit expressions for the screening energy and its gradient. J. Chem. Soc. Perkin Trans. 2 1993, 799-805.

(49) Cohen, A. J.; Mori-Sánchez, P.; Yang, W. Challenges for Density Functional Theory. Chem. Rev. 2012, 112, 289-320.

(50) Baer, R.; Livshits, E.; Salzner, U. Tuned Range-Separated Hybrids in Density Functional Theory. Ann. Rev. Phys. Chem. 2010, 61, 85-109. 
(51) Tamblyn, I.; Refaely-Abramson, S.; Neaton, J. B.; Kronik, L. Simultaneous Determination of Structures, Vibrations, and Frontier Orbital Energies from a Self-Consistent RangeSeparated Hybrid Functional. J. Phys. Chem. Lett. 2014, 5, 2734-2741.

(52) Autschbach, J.; Srebro, M. Delocalization error and 'functional tuning' in Kohn-Sham calculations of molecular properties. Acc. Chem. Res. 2014, 47, 2592-2602.

(53) Körzdörfer, T.; Brédas, J.-L. Organic Electronic Materials: Recent Advances in the DFT Description of the Ground and Excited States Using Tuned Range-Separated Hybrid Functionals. Acc. Chem. Res. 2014, 47, 3284-3291.

(54) Adamo, C.; Barone, V. Toward chemical accuracy in the computation of NMR shieldings: the PBE0 model. Chem. Phys. Lett. 1998, 298, 113-119.

(55) Shen, C.; Loas, G.; Srebro-Hooper, M.; Vanthuyne, N.; Toupet, L.; Cador, O.; Paul, F.; López Navarrete, J. T.; Ramírez, F. J.; Nieto-Ortega, B. et al. Iron Alkynyl Helicenes: Redox-Triggered Chiroptical Tuning in the IR and Near-IR Spectral Regions and Suitable for Telecommunications Applications. Angew. Chem. Int. Ed. 2016, 55, 8062-8066.

(56) Saleh, N.; Vanthuyne, N.; Bonvoisin, J.; Autschbach, J.; Srebro-Hooper, M.; Crassous, J. Redox-triggered chiroptical switching activity of ruthenium(III)-bis-( $\beta$-diketonato) complexes bearing a bipyridine-helicene ligand. Chirality 2018, 30, 592-601.

(57) Shen, C.; Srebro-Hooper, M.; Weymuth, T.; Krausbeck, F.; Lopez Navarrete, J. T.; Ramirez, F. J.; Nieto-Ortega, B.; Casado, J.; Reiher, M.; Autschbach, J.; Crassous, J. Redox-active Chiroptical Switching in Mono- and Bis-Iron-Ethynyl-Carbo[6]Helicenes Studied by Electronic and Vibrational Circular Dichroism and Resonance Raman Optical Activity. Chem. Eur. J. 2018, 24, 15067-15079.

(58) Ballester, M.; Riera-Figueras, J.; Castaner, J.; Badfa, C.; Monso, J. M. Inert carbon free radicals. I. Perchlorodiphenylmethyl and perchlorotriphenylmethyl radical series. J. Am. Chem. Soc. 1971, 93, 2215-2225.

(59) Ballester, M. Inert free radicals (IFR): a unique trivalent carbon species. Acc. Chem. Res. 1985, 18, 380-387.

(60) Heckmann, A.; Dümmler, S.; Pauli, J.; Margraf, M.; Köhler, J.; Stich, D.; Lambert, C.; Fischer, I.; Resch-Genger, U. Highly Fluorescent Open-Shell NIR Dyes: The TimeDependence of Back Electron Transfer in Triarylamine-Perchlorotriphenylmethyl Radicals. J. Phys. Chem. C 2009, 113, 20958-20966. 
(61) Amthor, S.; Noller, B.; Lambert, C. UV/Vis/NIR spectral properties of triarylamines and their corresponding radical cations. Chem. Phys. 2005, 316, 141-152.

(62) Bhosale, S. V.; Jani, C. H.; Langford, S. J. Chemistry of naphthalene diimides. Chem. Soc. Rev. 2008, 37, 331-342.

(63) Guha, S.; Goodson, F. S.; Corson, L. J.; Saha, S. Boundaries of Anion/Naphthalenediimide Interactions: From Anion- $\pi$ Interactions to Anion-Induced Charge-Transfer and ElectronTransfer Phenomena. J. Am. Chem. Soc. 2012, 134, 13679-13691 PMID: 22686833.

(64) Jones, B. A.; Facchetti, A.; Wasielewski, M. R.; Marks, T. J. Tuning Orbital Energetics in Arylene Diimide Semiconductors. Materials Design for Ambient Stability of n-Type Charge Transport. J. Am. Chem. Soc. 2007, 129, 15259-15278.

(65) Zhan, X.; Facchetti, A.; Barlow, S.; Marks, T. J.; Ratner, M. A.; Wasielewski, M. R.; Marder, S. R. Rylene and Related Diimides for Organic Electronics. Adv. Mater. 2011, 23, 268-284.

(66) Sakai, N.; Mareda, J.; Vauthey, E.; Matile, S. Core-substituted naphthalenediimides. Chem. Commun. 2010, 46, 4225-4237.

(67) Shybeka, I.; Aster, A.; Cheng, Y.; Sakai, N.; Frontera, A.; Vauthey, E.; Matile, S. Naphthalenediimides with Cyclic Oligochalcogenides in Their Core. Chem. Eur. J. 2020, 26, 14059-14063.

(68) Shukla, J.; Mukhopadhyay, P. Synthesis of Functionalized Naphthalene Diimides and their Redox Properties. Eur. J. Org. Chem. 2019, 2019, 7770-7786.

(69) Center for Computational Research, University at Buffalo. URL http: / / hdl . handle. net/10477/79221. Accessed 11/2019. 


\section{for Table of Contents use only}

Why is the energy of the singly occupied orbital in some radicals below the highest occupied orbital energy?

Laura Abella, ${ }^{a}$ Jeanne Crassous, ${ }^{b}$ Ludovic Favereau ${ }^{b}$, and Jochen Autschbach ${ }^{a, *}$

${ }^{a}$ Department of Chemistry, University at Buffalo, State University of New York, Buffalo, NY

14260-3000, USA. Fax: (+) +01-716-645-6963. email: jochena@buffalo.edu

${ }^{b}$ Univ Rennes, CNRS, ISCR - UMR 6226, F-35000 Rennes, France

$$
\varepsilon_{i, \sigma}=T_{i, \sigma}+V_{i, \sigma}^{n u c}+V_{i, \sigma}^{C}+V_{i, \sigma}^{X C}
$$

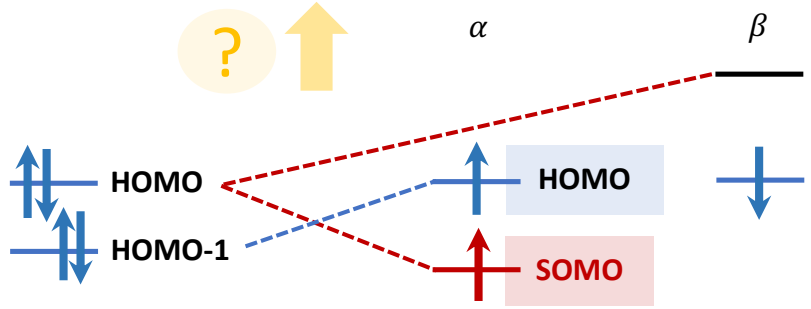

Closed-Shell $\longrightarrow$ Radical with SHI 